\title{
Redundant Function of REV-ERB $\alpha$ and $\beta$ and Non-Essential Role for Bmal1 Cycling in Transcriptional Regulation of Intracellular Circadian Rhythms
}

\author{
Andrew C. Liu ${ }^{1,2}$, Hien G. Tran ${ }^{1,2}$, Eric E. Zhang ${ }^{1,2}$, Aaron A. Priest ${ }^{1}$, David K. Welsh ${ }^{1,3,4}$, Steve A. Kay ${ }^{1 *}$ \\ 1 Section of Cell and Developmental Biology, Division of Biological Sciences, University of California San Diego, La Jolla, California, United States of America, 2 Genomics \\ Institute, Novartis Research Foundation, San Diego, California, United States of America, 3 Department of Psychiatry, University of California San Diego, La Jolla, California, \\ United States of America, 4 Veterans Affairs, San Diego Healthcare System, San Diego, California, United States of America
}

\begin{abstract}
The mammalian circadian clockwork is composed of a core PER/CRY feedback loop and additional interlocking loops. In particular, the ROR/REV/Bmal1 loop, consisting of ROR activators and REV-ERB repressors that regulate Bmal1 expression, is thought to "stabilize" core clock function. However, due to functional redundancy and pleiotropic effects of gene deletions, the role of the ROR/REV/Bmal1 loop has not been accurately defined. In this study, we examined cell-autonomous circadian oscillations using combined gene knockout and RNA interference and demonstrated that REV-ERB $\alpha$ and $\beta$ are functionally redundant and are required for rhythmic Bmal1 expression. In contrast, the RORs contribute to Bmal1 amplitude but are dispensable for Bmal1 rhythm. We provide direct in vivo genetic evidence that the REV-ERBs also participate in combinatorial regulation of Cry 1 and Rorc expression, leading to their phase-delay relative to Rev-erb $\alpha$. Thus, the REV-ERBs play a more prominent role than the RORs in the basic clock mechanism. The cellular genetic approach permitted testing of the robustness of the intracellular core clock function. We showed that cells deficient in both REV-ERB $\alpha$ and $\beta$ function, or those expressing constitutive BMAL1, were still able to generate and maintain normal Per2 rhythmicity. Our findings thus underscore the resilience of the intracellular clock mechanism and provide important insights into the transcriptional topologies underlying the circadian clock. Since REV-ERB function and Bmal1 mRNA/protein cycling are not necessary for basic clock function, we propose that the major role of the ROR/REV/Bmal1 loop and its constituents is to control rhythmic transcription of clock output genes.
\end{abstract}

\footnotetext{
Citation: Liu AC, Tran HG, Zhang EE, Priest AA, Welsh DK, et al. (2008) Redundant Function of REV-ERB $\alpha$ and $\beta$ and Non-Essential Role for Bmal1 Cycling in Transcriptional Regulation of Intracellular Circadian Rhythms. PLoS Genet 4(2): e1000023. doi:10.1371/journal.pgen.1000023

Editor: Joseph S. Takahashi, Howard Hughes Medical Institute, Northwestern University, United States of America

Received September 10, 2007; Accepted December 28, 2007; Published February 29, 2008

Copyright: $\odot 2008$ Liu et al. This is an open-access article distributed under the terms of the Creative Commons Attribution License, which permits unrestricted use, distribution, and reproduction in any medium, provided the original author and source are credited.

Funding: This work was supported in part by grants from the NIH (R01 GM074868 and R01 MH051573 to SAK, K08 MH067657 to DKW). This is manuscript \#19080 of The Scripps Research Institute and manuscript \#070804 of the Genomics Institute of the Novartis Research Foundation.

Competing Interests: The authors have declared that no competing interests exist.

*E-mail: skay@ucsd.edu
}

\section{Introduction}

Circadian rhythms in physiology and behavior are regulated by endogenous circadian clocks. All the molecular clocks so far described in multicellular organisms constitute negative feedback loops in which protein products of clock genes inhibit transcription of their own genes [1]. In mammals, the central pacemaker in the suprachiasmatic nuclei (SCN) integrates light-dark cycle input and coordinates oscillators in peripheral tissues [2]. Like the SCN, peripheral tissues also contain cell-autonomous circadian oscillators. The current cellular clock model comprises a core feedback loop consisting of PER and CRY repressors and BMAL1 and CLOCK activators $[1,3]$. In the core loop, BMAL1/CLOCK heterodimers directly bind to E-box enhancer elements present in $\operatorname{Per}(\operatorname{Per} 1$ and Per2) and Cry (Cry1 and Cry2) genes and activate their transcription; PER and CRY proteins in turn repress their own transcription through direct interactions with BMAL1/CLOCK.

The mammalian clock has been shown to contain additional interlocking loops. In particular, the ROR/REV/Bmal1 feedback loop consists of the RORs (RORa, b and c) and REV-ERBs (REV-ERB $\alpha$ and $\beta$ ), members of a subfamily of orphan nuclear receptors [4], whose expression is directly regulated by the core loop [5-8]. To drive rhythmic expression of Bmal1, REV-ERB $\alpha$ represses Bmall transcription by directly binding to the ROR elements (ROREs) in the Bmal1 promoter [5,9]; RORa and RORb, on the other hand, act as positive drivers to activate Bmall expression in the SCN [6,9-11]. The roles of REV-ERB $\beta$ and RORc in clock function have not been addressed.

An analogous set of interlocking loops has been described in the Drosophila circadian clock $[7,12,13]$. The dPER/dTIM repressors and dCLK/dCYC activators constitute the core feedback loop. In the interlocked $d C l k$ feedback loop, the bZIP transcription factors dPDP1 and dVRI, which are directly controlled by the core loop, activate and repress $d C l k$ transcription, respectively. However, unlike the requirement for cyclic expression of $d P e r$ and $d T$ im mRNAs, it was shown that $d C l k$ mRNA cycling is not necessary for molecular and behavioral rhythms in flies [14-16]. The $d C l k$ loop function in flies could not be precisely defined genetically, however, because mutants deficient in $d V r i$ and/or $d P d p$ are developmentally lethal $[12,16]$.

The role of the ROR/REV/Bmal1 loop in mammals has not been precisely addressed either, due to functional redundancy of 


\section{Author Summary}

Circadian clocks in plants, fungi, insects, and mammals all share a common transcriptional network architecture. At the cellular level, the mammalian clockwork consists of a core Per/Cry negative feedback loop and additional interlocking loops. We wished to address experimentally the contribution of the interlocking Bmal1 loop to clock function in mammals. Because behavioral rhythms do not always reflect cell-autonomous phenotypes and are subject to pleiotropic effects, we employed cell-based genetic approaches and monitored rhythms longitudinally using bioluminescent reporters of clock gene expression. We showed that REV-ERB repressors play a more prominent role than ROR activators in regulating the Bmal1 rhythm. However, significant rhythmicity remains even with constitutive expression of Bmal1, pointing to the resilience of the core loop to perturbations of the Bmal1 loop. We conclude that while the interlocking loop contributes to fine-tuning of the core loop, its primary function is to provide discrete waveforms of clock gene expression for control of local physiology. This study has important general implications not only for circadian biology across species, but also for the emerging field of systems biology that seeks to understand complex interactions in genetic networks.

the RORs and REV-ERBs and pleiotropic effects of gene deletions. As deletion of Rev-erb $\alpha$, Rora or Rorb results in a broader distribution of circadian period lengths, it was suggested that the ROR/REV/Bmal1 loop serves as a stabilizing mechanism [5$7,10]$. However, Ror mutant mice exhibit potentially confounding non-circadian phenotypes. Rorb ${ }^{-/-}$mice display reproductive deficits and a severe postnatal retinal degeneration [10]. Rora knockout $\left(\right.$ Rora $\left.^{-/-}\right)$and mutant staggerer $\left(\right.$Rora $\left.^{\text {sg/sg }}\right)$ mice display cerebellar ataxia and are mostly infertile $[6,11,17,18]$. Importantly, period dispersion is not unique to animals deficient in Ror or Rev-erb function; Per1 $1^{-/-}$, Per2 $2^{-/-}$and $C l o c k^{m / m}$ mice also display less persistent circadian behavior and larger variability of periods [19-22]. Thus, circadian abnormalities in these mice measured using behavioral outputs may not faithfully reflect intracellular clock function. Finally, functional redundancy cannot be addressed genetically at the behavioral level because compound knockout animals have gross defects.

The drawbacks of behavioral analysis can be circumvented by studies using cell-based clock models. Strategically, molecular mechanisms required for rhythmicity are best studied at the cellular level using long-term recordings to assess persistence of circadian rhythmicity [23]. In this study, by taking advantage of a cell-based experimental model and real-time bioluminescence monitoring of gene expression, we first define the roles of RORc and REV-ERB $\beta$ in peripheral clock function, and then extend our studies to include all the RORs and REV-ERBs and their respective contributions to circadian rhythms of Bmal1 expression. Furthermore, we show that the REV-ERBs are necessary for Bmal1 rhythm while the RORs are dispensable, indicating that the REV-ERBs play a more prominent role than the RORs in the transcriptional circuitry of the clockwork. Importantly, however, rhythmic Bmal1 mRNA and protein expression is not required for the basic operation of the core clock. These results are in line with the observation that constitutive Bmall expression was able to rescue circadian behavioral rhythms in Bmal1 $^{-/-}$mice [24]. We suggest that the major role of the ROR/REV/Bmall loop is to provide additional phase modulation for establishing transcriptional output networks.

\section{Results}

\section{Differential Tissue Distribution of the Rors}

We first examined expression of the Rors and Rev-erbs in various tissues (Figure S1A, S1B, S1C). In contrast to the ubiquitous expression of Bmal1, Rev-erb $\alpha$ and Rev-erbb in all the tissues examined, expression and rhythmicity of the RORs are more restrictive. Rora expression is ubiquitous, but its circadian cycling is restricted to SCN. Rorb is expressed in the SCN, hypothalamus, cerebral cortex and retina, but not in the liver. Conversely, Rorc is rhythmically expressed in the liver, but not detected in the SCN or other brain regions. Expression patterns of the Ror genes in the lung were similar to those in the liver (data not shown). The tissuespecific expression patterns of the RORs are consistent with previous reports [6,25-28].

In this study, we extensively used fibroblasts derived from mice as a cell-based clock model. Of the three Rors, only Rora is highly expressed in mouse fibroblasts, but no distinct mRNA rhythm was detected (Figure S1A); Rorb and Rorc were not detected in fibroblasts (Figure S1A and S1B). Differential tissue distribution and rhythmicity of the Rors suggests that they may have different functions in clock mechanisms.

\section{Rorc $^{-\prime-}$ Mice Display Normal Circadian Rhythms}

Rora and Rorb have been characterized as clock components, functioning to regulate Bmal1 expression in the SCN (Figure S1C) $[6,10,11,25]$. However, since RORc is not expressed in the SCN, it should not affect function of the SCN pacemaker, which drives circadian locomotor behavior. We tested this hypothesis in a mouse line deficient in Rorc function. Deletion of Rorc results in reduced survival of thymocytes and abnormal lymphoid organ development, but $B c l-x L$ transgene $\left(B c l-x L^{T g}\right)$ expression restored most aspects of normal thymocyte development and significantly improved animal survival [29]. Compared to $B c l-x L^{T g}$ control (period length $\tau=23.42 \mathrm{hr} \pm 0.08, \mathrm{n}=5$ ), Rorc $^{-1-}: B c l-x L^{T_{g}}$ mice displayed normal circadian wheel-running activity under constant darkness $(\tau=23.34 \mathrm{hr} \pm 0.2, \mathrm{n}=8)$. These mice also showed a normal response to a light pulse at CT16 (Figure S1D). We further examined the dynamics of molecular rhythms in the SCN and showed that SCN explants from $\operatorname{Rorc}^{-1-}: B c l-x L^{T g}$ mice also displayed similar mPer2 ${ }^{\text {Luc }}$ bioluminescence rhythms to control mice (data not shown). Thus, consistent with the absence of Rorc gene expression in the SCN, these results confirm that RORc plays no role in SCN pacemaker function.

\section{RORc Regulates Circadian Bmal1 Transcription in the Liver}

Based on the ability of RORc to activate a Bmal1-Luc reporter in vitro and its strong rhythmic expression in many peripheral tissues including the liver and lung $[6,9,25]$, we hypothesized that RORc, like RORa and RORb in the SCN, may play an important role as an activator of Bmal1 in peripheral oscillators. We tested this hypothesis by analyzing Bmal1 expression in the mouse liver. In $B c l-x L^{T g}$ control mice, Bmall expression peaked at CT24 (Figure 1A). In contrast, Bmal1 expression at CT28, CT44 and CT48 in the liver of $\operatorname{Rorc}^{-1-}: B c l-x L^{T g}$ mice was significantly reduced, compared to those of $B c l-x L^{T g}$ siblings (Figure 1A). These results suggest that RORc activates Bmal1 transcription in the positive arm of the ROR/REV/Bmall loop, functioning to maintain normal amplitude of Bmal1 rhythmicity.

Although Bmal1 peak expression levels are reduced in the absence of RORc, Bmal1 mRNA still retains a rhythm with fairly high amplitude, indicative of functional redundancy from RORa and/or contributions from the REV-ERBs. RORc also regulates 

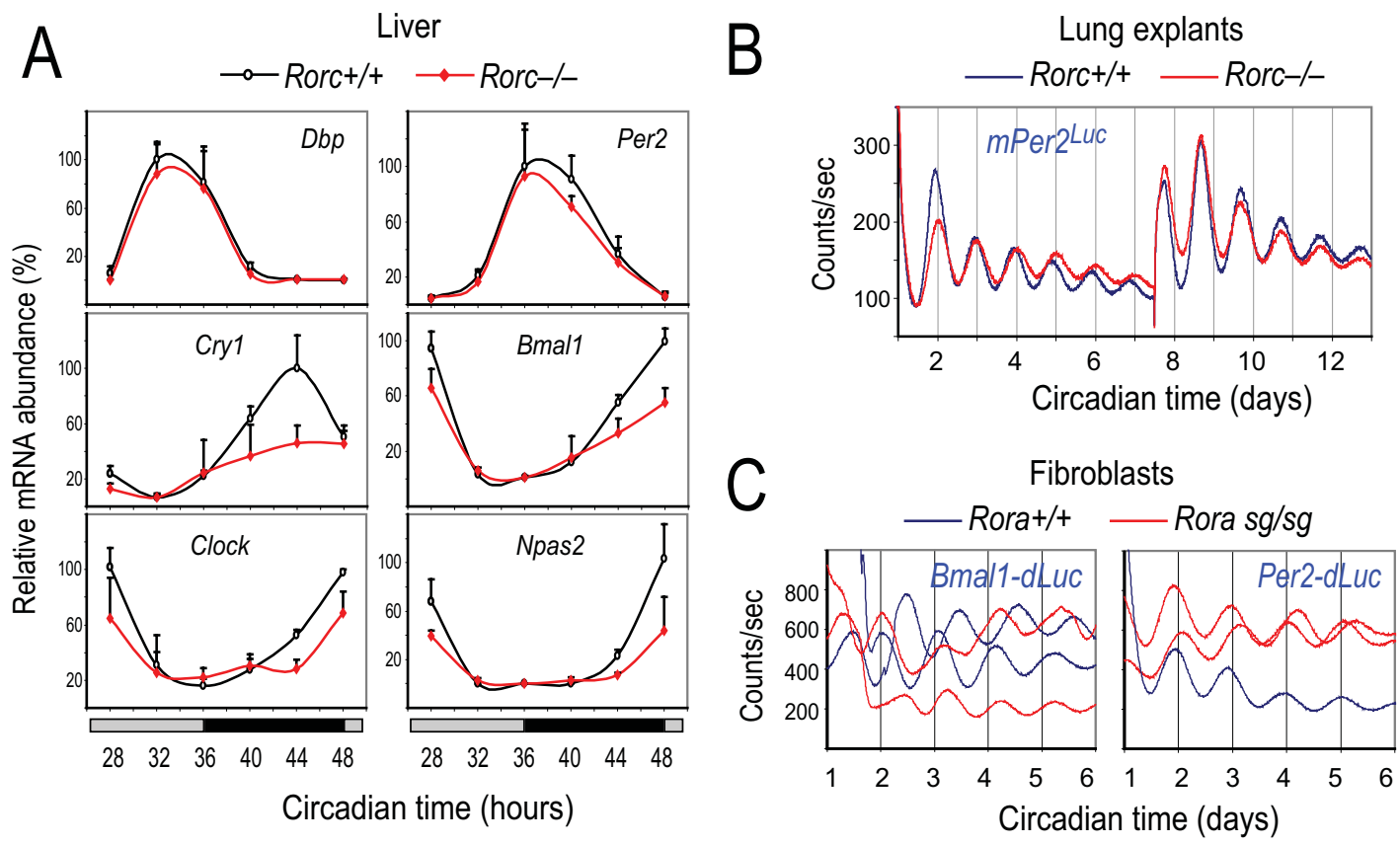

Figure 1. RORc activates Bmal1 transcription but is dispensable for Bmal1 rhythmicity. (A) Circadian Bmal1 mRNA expression is blunted in the liver of Rorc ${ }^{-1-}$ mice. The peak transcript levels of Bmal1, Clock, Npas2, and Cry1 are reduced in the liver of Rorc ${ }^{-1-}$ mice compared to WT littermates, suggesting that RORc is an activator of Bmal1 transcription. Temporal patterns of Per2 and Dbp are unaltered. Expression was analyzed at 4-hr intervals by Q-PCR. Values are expressed as percentage of maximum expression for each gene. Error bars represent standard deviation (SD) of expression levels from four mice. Circadian time: hours after animal release in constant darkness. (B) Representative records of tissue-autonomous mPer2 $2^{\text {Luc }}$ bioluminescence rhythms in Rorc ${ }^{-1-}$ lung explants. Rorc ${ }^{-1-}$ lung explants displayed normal mPer2 ${ }^{\text {Luc }}$ rhythms, suggesting that Rorc is not required for circadian rhythmicity. Tissue explants were dissected and immediately cultured in explant medium for recording. Another medium change occurred at day 7. Circadian time: days after explant medium change. (C,D) Representative records of cell-autonomous bioluminescence rhythms in populations of Rora ${ }^{s g / s g}$ fibroblasts transduced with a lentiviral Bmal1-dLuc reporter (C) or Per2-dLuC reporter (D). Rora ${ }^{5 g / s g}$ fibroblasts, in which no functional RORa, RORb, or RORc are expressed, displayed rhythmic oscillations of Bmal1-dLuc and Per2-dLuc reporters. Circadian time: days after explant medium change.

doi:10.1371/journal.pgen.1000023.g001

transcription of Cry 1, Clock and Npas2, all of which are considered RORE-containing genes [30,31], and their mRNAs were also reduced during peaking hours (Figure 1A). Despite the blunted rhythm amplitudes for Bmal1, Clock, Npas2 and Cry1, cyclic expression of Per2 and $D b p$, however, was not dramatically affected by Rorc deletion, similar to observations in Rev-erb $\alpha^{-/-}$mice [5].

\section{RORc Is Not Required in Peripheral Clock Function}

As the SCN clock functions normally in the absence of Rorc, we assessed the effect of Rorc deletion on peripheral clock function in tissue-autonomous preparations in which confounding influences from the SCN are eliminated. Tissue explants of the lung from $\mathrm{Bcl}$ $x L^{T g}$ control mice displayed persistent mPer2 ${ }^{\text {Luc }}$ rhythms $(\tau=24.00 \mathrm{hr} \pm 0.33, \mathrm{n}=4)$. Rorc ${ }^{-1-}: B c l-x L^{T g}$ lung explants exhibited rhythmic $m P e r 2^{L u c}$ expression with comparable period lengths to controls $(\tau=24.15 \pm 0.49, \mathrm{n}=4)$ (Figure 1B). Rorc $^{-1-}: B c l-x L^{T g}$ liver explants also displayed persistent mPer2 ${ }^{L u c}$ rhythms $(\tau=22.59 \mathrm{hr} \pm 1.54, \quad \mathrm{n}=5), \quad$ similar to controls $(\tau=22.22 \mathrm{hr} \pm 0.71, \mathrm{n}=3$ ). Surprisingly, no significant differences in circadian amplitude or damping rate were observed between controls and Rorc $^{-/-}$mice. The normal bioluminescence rhythms are consistent with unaltered molecular phenotypes of Per2 expression (Figure 1A). Moreover, we observed normal rhythms in fibroblasts, in which Rorc expression is not detectable (data not shown), further confirming results from liver and explants. In fibroblasts, over-expression of Rorc did not affect Bmall rhythms (data not shown). These results demonstrate that RORc does not play an essential role in maintaining circadian oscillation and suggest that a high-amplitude Bmal1 rhythm may not be critically required for basic clock operation, similar to phenotypes observed for Rev-erb $\alpha$ deficiency [5].

\section{The ROR Activators Are Not Required for Bmal1 Rhythmicity in Fibroblasts}

So far, data suggest a functional redundancy among RORa, RORb and RORc. In the liver and fibroblasts of both Rora ${ }^{s g / s g}$ [6,11] and Rorc $^{-/-}$mice, Bmal1 peak expression is reduced, but the mRNA rhythm is largely retained and Per2 oscillation is not altered. Although Rora does not show strong rhythmicity in the liver, its expression alone could partially complement the loss of Rorc. To study the ROR redundancy genetically, a mouse line deficient in both Rora and Rorc would represent an ideal reagent. However, such a line is extremely difficult to obtain because Rora ${ }^{s g} /$ ${ }^{s g}$ mutant mice display cerebellar ataxia and mostly infertile [18] and Rorc $^{-1-}$ mice also have strongly abnormal phenotypes [29]. Therefore, we decided to address the ROR redundancy using Rora ${ }^{s g / s g}$ fibroblasts. Because Rorb and Rorc are also not expressed in Rora $^{s g / s g}$ fibroblasts as determined by Q-PCR (data not shown), thus excluding the possibility of a compensation mechanism, the positive arm of the ROR/REV/Bmal1 loop is essentially missing in cells lacking Rora function.

To monitor the function of the core loop and the ROR/REV/ Bmal1 loop in parallel, we generated two lentivirus-mediated circadian reporters, $p L V 6-P e r 2-d L u c$ and $p L V 6-B m a l 1-d L u c$, designed to report the E-box and RORE-regulated rhythms, 
respectively. As expected, WT cells displayed persistent Bmal1-dLuc rhythms $(\tau=24.44 \pm 1.55 \mathrm{hr}, \mathrm{n}=17$ culture dishes from 2 independent cell lines). Importantly, Rora ${ }^{s g / s g}$ fibroblasts also displayed rhythmic Bmal1-dLuc oscillations $(\tau=24.34 \pm 0.95 \mathrm{hr}$, $\mathrm{n}=30$ from 3 lines), comparable to WT cells (Figure 1C). Not surprisingly, these cells also exhibited Per2- $d L u c$ rhythms similar to those of WT cells (Figure 1G). Our results demonstrate that the ROR activators contribute to Bmall rhythm amplitude, but are clearly not required for Bmal1 rhythmicity and core clock function in fibroblasts.

\section{REV-ERB $\alpha$ and $\beta$ Are Required for Bmal1 Rhythmicity in Fibroblasts}

Next, we examined the consequence of disrupting the negative arm of the ROR/REV/Bmal1 loop. Bmall expression is significantly higher in the liver [5] and fibroblasts of Rev-erb $\alpha^{-1-}$ mice than in WT (data not shown). Given the abnormal Bmal1 expression in the liver and fibroblasts, we expected that deletion of Rev-erb $\alpha$ would dramatically compromise the Bmal1 rhythm, as previously suggested from mRNA analysis [5]. Surprisingly, however, Rev-erb $\alpha^{-/-}$fibroblasts displayed rhythmic Bmal1-dLuc expression (Figure 2A). The period lengths for Rev-erb $\alpha^{-1-}$ fibroblasts harboring Per2-dLuc reporter were determined to be $26.59 \pm 0.29 \mathrm{hr}(\mathrm{n}=10)$ for cell line-1 and 24.25 $\pm 0.72 \mathrm{hr}(\mathrm{n}=10)$ for cell line-2, and the corresponding WT fibroblasts exhibited a periodicity of $24.88 \pm 1.48 \mathrm{hr}(\mathrm{n}=7)$. Thus, as expected, real-time longitudinal bioluminescence recording reveals the dynamics of gene expression, while mRNA profiling lacks temporal resolution and is generally more subject to noise. Given the apparent redundant contribution from Rev-erbb, Bmal1 rhythms in the liver and lung of Rev-erb $\alpha^{-1-}$ mice are also likely to be rhythmic, similar to that observed in fibroblasts.

We assessed any redundant contribution from Rev-erbb using small hairpin RNAs (shRNA). We designed and tested nine shRNA constructs against different regions of the Rev-erbb gene, and three of them (shRNA- $\beta 1, \beta 2$ and $\beta 3$ ) were found to be functional in efficiently knocking down Rev-erbb expression (Figure 2C). We introduced Rev-erbb-shRNA constructs into WT fibroblasts harboring Bmal1-dLuc reporter. Knockdown of Rev-erbb resulted in higher Bmal1 mRNA expression, with shRNA- $\beta 1$ being the most potent (Figure 2C); these cells displayed rhythmic Bmal1$d$ Luc expression (Figure 2A), similar to effects of Rev-erb $\alpha$-knockout. Thus, Rev-erb $\alpha$ and Rev-erbb are functionally redundant and disruption of either one alone is not sufficient to disrupt Bmal1 rhythms.

To disrupt the function of $\operatorname{REV}-\mathrm{ERB} \alpha$ and $\beta$ simultaneously, Rev-erbb-shRNA constructs were stably introduced into Rev-erb $\alpha^{-/-}$ fibroblasts harboring the Bmal1-dLuc reporter to obtain Rev$e r b \alpha^{-1-}:$ Rev-erbb-shRNA:Bmal1-dLuc cell lines. In striking contrast to rhythmic Bmal1-dLuc expression in Rev-erb $\alpha$-knockout or Reverbb-knockdown fibroblasts, cells deficient in both Rev-erb $\alpha$ and $b$ function displayed significantly higher levels but largely arrhythmic Bmal1-dLuc expression (Figure 2B). For cell line-2, 15/18 dishes of Rev-erb $\alpha^{-1-}$ cells expressing control shRNA displayed rhythmic Bmal1-dLuc expression (FFT spectral amplitude $=0.80 \pm 0.08, \mathrm{n}=15)$, but only 6/19 of Rev-erb $\alpha^{-\prime-}$ :Rev-erbbshRNA- $\beta 1$ showed any rhythms, and those that were rhythmic showed significantly lower spectral amplitude (FFT spectral amplitude $=0.50 \pm 0.08, \mathrm{n}=6)$. The weak rhythms may likely result from residual levels of REV-ERB $\beta$ expression in these knockdown cells. Similar results were observed in cell line-1 (data not shown). These results demonstrate that the REV-ERB $\alpha$ and $\beta$ are required for rhythmic Bmal1 expression in fibroblasts. The finding that cells lacking ROR function retain Bmal1-dLuc rhythms whereas those deficient in REV-ERB function are arrhythmic, suggests that the REV-ERB repressors play more prominent roles than the ROR activators in the ROR/REV/ Bmal1 loop.

\section{Rev-erb $\alpha$ and $\beta$ Are Not Required for PER/CRY Core Loop Function}

Given that the Bmal1-dLuc reporter is rhythmic in Rev-erb $\alpha^{-/-}$ fibroblasts, it is not surprising to observe that the Per2-dLuc reporter was also rhythmic (Figure 2D). However, it was not known whether disrupting both Rev-erb $\alpha$ and $b$ would affect the core feedback loop function. We thus introduced Rev-erbbshRNA constructs into Rev-erb $\alpha^{-/-}:$Per2-dLuc fibroblasts and

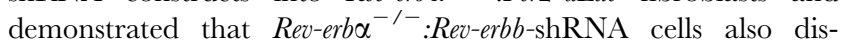
played rhythmic patterns of Per2-dLuc expression $(\tau=25.99 \pm$ $0.40 \mathrm{hr}, \mathrm{n}=7$ for cell line- $1 ; \tau=25.12 \pm 0.60 \mathrm{hr}, \mathrm{n}=22$ for cell line-2), similar to cells expressing control shRNA $(\tau=26.48 \pm 0.27 \mathrm{hr}, \mathrm{n}=7$ for cell line-1; $\tau=25.31 \pm 0.52 \mathrm{hr}$, $\mathrm{n}=23$ for cell line-2) (Figure 2D).

We also examined effects of Rev-erbb-knockdown on the expression of other clock genes. In shRNA control cells, peaks of Rev-erbb and Per2 mRNAs (CT40-48) were almost anti-phasic to Bmal1 (CT32-36). Bmal1 mRNA was effectively de-repressed, especially at CT46-52 when Bmal1 was at its nadir in control cells (Figure 2C). Consistent with rhythmic Per2-dLuc bioluminescence expression, the Per 2 mRNA expression pattern was essentially the same in Rev-erb $\alpha^{-1-}$ cells expressing control shRNA and in those expressing shRNA against Rev-erbb.

Given that Cry 1 is under combinatorial regulation by both BMAL1/CLOCK and REV-ERBs [5,30,31], we expected that disruption of REV-ERB function would alter the Cry 1 expression pattern. Indeed, compared to WT cells, Cry $1 \mathrm{mRNA}$ levels were higher in Rev-erb $\alpha^{-1-}$ fibroblasts (data not shown), and even

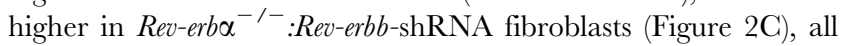
consistent with REV-ERB proteins being repressors. Although interference with the REV-ERBs clearly disrupted the Bmal1 rhythm, it did not seem to substantially alter the rhythm of Cry 1 mRNA. Cry 1 mRNA remained to be rhythmic, reaching its nadir at CT36-40 and peaking at CT46-50, illustrating the resilience of the intracellular clock mechanism. It is possible that, even though the Bmal1 rhythm is abolished, the residual level of REV-ERB $\beta$ in the cells was sufficient for combinatorial regulation of Cry1. It is also possible that other unknown mechanisms contribute to Cry 1 regulation. This ambiguity can be resolved in future studies by examining cells completely deficient in both Rev-erb $\alpha$ and $b$ function. Nevertheless, our results suggest that REV-ERB $\alpha$ and $\beta$ are required for rhythmic expression of Bmal1, but REV-ERB function and the Bmal1 rhythm are not required for normal oscillations of Per and Cry.

\section{Constitutive BMAL1 Restores Circadian Rhythmicity in Bmal1 $^{-/-}$Fibroblasts}

To further test the role of RORE-mediated Bmal1 regulation, we eliminated all influences of the RORs and REV-ERBs on Bmal1 expression in cell-based genetic complementation experiments. Fibroblasts derived from Bmal1 ${ }^{-/-}: m P e r 2^{L u c}$ mice displayed arrhythmic patterns of bioluminescence expression, demonstrating that Bmal1 is an essential clock component for cellular rhythmicity in fibroblasts (Figure 3A). We asked whether constitutively expressed BMAL1 in Bmal1 ${ }^{-/-}$fibroblasts could restore circadian rhythmicity. This approach precludes residual REV-ERB $\beta$ function from shRNA knockdown and circumvents any off-target effects. 

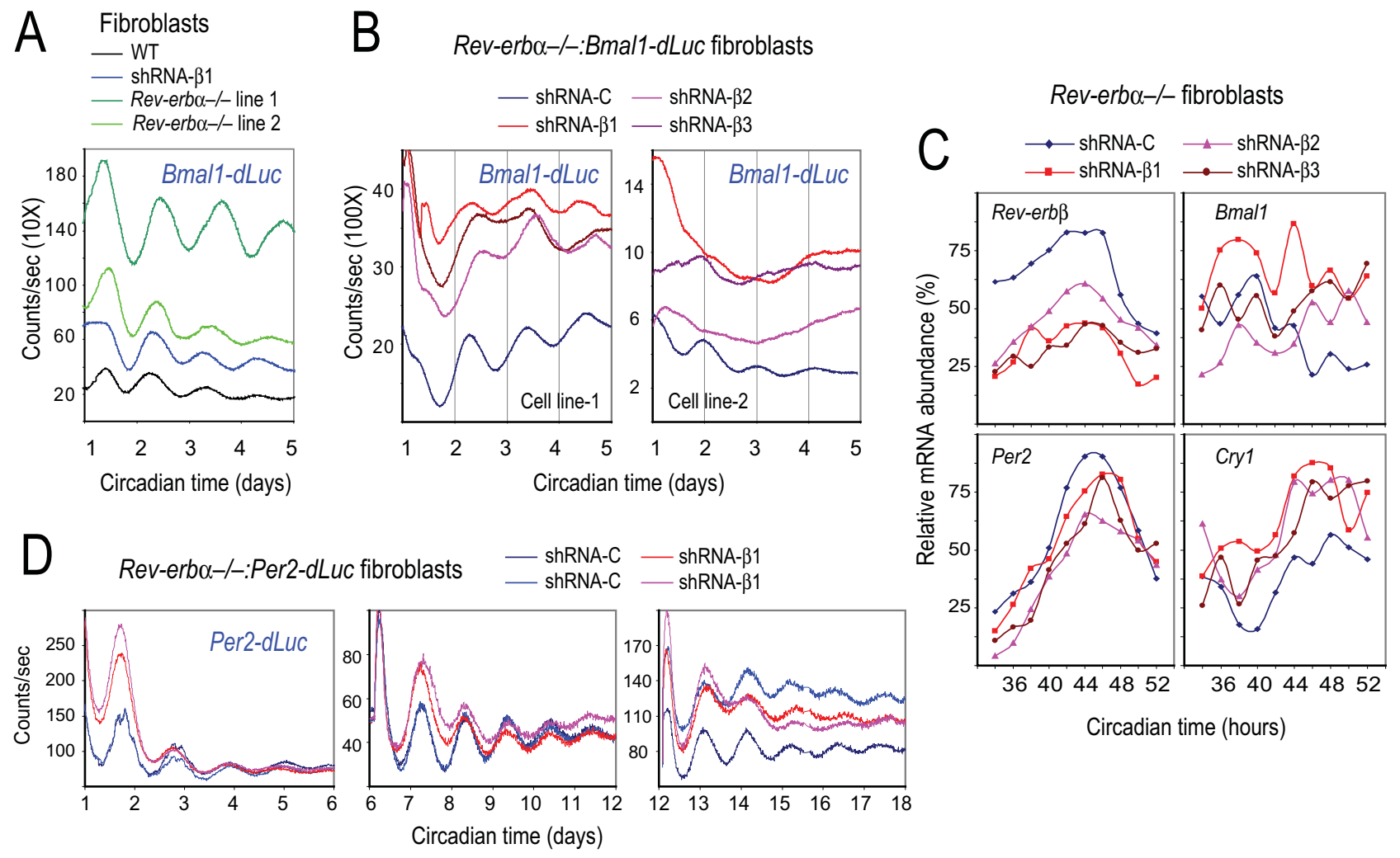

Figure 2. REV-ERB $\alpha$ and $\beta$ are required for Bmal1 rhythms in fibroblasts. (A) Representative bioluminescence rhythms from a Bmal1-dLuc reporter in fibroblasts deficient in either Rev-erb $\alpha$ or Rev-erb $\beta$ function. We tested two independent Rev-erb $\alpha^{-/-}$fibroblast cell lines and cells stably expressing an shRNA construct against Rev-erb $\beta$. Fibroblasts deficient in either Rev-erb $\alpha$ or Rev-erb $\beta$ function alone displayed rhythmic oscillations of Bmal1-dLuc bioluminescence, suggesting functional redundancy of Rev-erb $\alpha$ and Rev-erb $\beta$. Circadian time: days after explant medium change. (B) Representative bioluminescence patterns from a Bmal1-dLuc reporter in fibroblasts deficient in both Rev-erb $\alpha$ and Rev-erb $\beta$ function. Rev-erb ${ }^{-1-}$ fibroblasts stably expressing a non-specific control shRNA (shRNA-C) displayed circadian Bmal1-dLuc rhythms, but Rev-erb $\alpha^{-1-}$ :Rev-erb $\beta$-shRNA cells were arrhythmic, suggesting that the REV-ERBs are required for Bmal1 rhythmic expression. Three different shRNA constructs (shRNA- $\beta 1, \beta 2$ and $\beta 3$ ) were used for knocking down endogenous Rev-erb $\beta$ expression in fibroblasts. Circadian time: days after explant medium change. (C) Temporal mRNA expression profiles of clock genes in Rev-erb $\alpha^{-1-}$ fibroblasts stably expressing shRNA constructs against Rev-erb $\beta$. Expression was analyzed at 2-hr intervals by Q-PCR. Values are expressed as percentage of maximum expression for each gene. Results were confirmed in two independent time courses. Similar results were obtained from both cell lines, and results for cell line-2 are presented here. For clarity, error bars representing SD of two culture samples for each cell line $(<10 \%)$ were omitted. Rev-erb $\beta$ mRNA was significantly reduced by shRNA against Rev-erb $\beta$, leading to higher expression levels of Bmal1 and Cry1. Per2 mRNA rhythms were unaltered in cells deficient in Rev-erb $\alpha$ and $\beta$ function. Circadian time: hours after serum treatment. (D) Representative bioluminescence rhythms from a Per2-dLuc reporter in fibroblasts deficient in both Rev-erb $\alpha$ and Rev-erb $\beta$ function. Rev-erb $\alpha^{-1-}$ fibroblasts expressing shRNA constructs against Rev-erb $\beta$ displayed Per2-dLuc rhythms similar to those of shRNA control cells. Similar results were obtained from all three shRNA constructs in two Rev-erb $\alpha^{-1-}$ fibroblast cell lines, and results from cell line-2 are presented here. The three panels show patterns for the same cultures after three successive medium changes. Circadian time: days after explant medium change. doi:10.1371/journal.pgen.1000023.g002

To manipulate Bmal1 expression, we used three promoters: Bmal1(WT) contains a 526-bp DNA fragment from the Bmal1 promoter encompassing ROREs, Bmall(Mut) is identical to Bmal1(WT) except that the RORE sites are mutated to prevent ROR/REV-ERB from binding, and $U b C$ is a commonly used constitutive promoter from the $U b C$ gene. We showed that $\mathrm{WT}$ fibroblasts transduced with a lentiviral $\operatorname{Bmal1}(\mathrm{WT})-d L u c$ reporter displayed rhythmic bioluminescence expression, but Bmal1(Mut) or $U b C$ promoters did not confer rhythmicity in these cells (Figure 3B).

We next determined the ability of the promoters to regulate the expression of Bmal1. In lieu of Western blot analysis of BMAL1, we monitored the bioluminescence expression of BMAL1::LUC fusion protein. We demonstrated that BMAL1::LUG cycled only when it is driven by Bmal1(WT), and that $U b C$ and Bmal1(Mut) promoters did not confer rhythmic fusion protein expression (Figure 3C). Thus, BMALl protein itself does not cycle in the absence of a RORE-containing circadian promoter.
To carry out genetic complementation, we generated a lentiviral expression vector Bmal1(WT)-Bmal1·Flag, in which Bmal1 cDNA is under the control of WT Bmal1 promoter. When this construct was introduced into $\mathrm{Bmal1}^{-1-}: \mathrm{mPer} 2^{\text {luc }}$ fibroblasts, circadian rhythmicity was restored $(\tau=22.02 \pm 0.68 \mathrm{hr}, \mathrm{n}=25$ cultured dishes) (Figure 3D), but not in cells expressing a Bmal1(WT)-GFP control construct (data not shown). Importantly, non-cyclically expressed BMAL1 under the control of either $U b C$ or Bmal1(Mut) also effectively restored circadian $m P e r 2^{L u c}$ rhythmicity in Bmal1 ${ }^{-1-}$ fibroblasts $(\tau=22.08 \pm 0.46 \mathrm{hr}, \mathrm{n}=20$ for UbC-Bmal1; $\tau=22.61 \pm 0.60 \mathrm{hr}, \mathrm{n}=27$ for Bmal1(Mut)-Bmal1) (Figure 3D). Taken together, these results demonstrate that rhythmic expression of BMALl protein is not essential for the basic functioning of the intracellular clock. These results provide the cellular basis for the finding that constitutive Bmal1 expression was able to rescue circadian behavioral rhythms in Bmall $^{-/-}$mice $[24]$. 

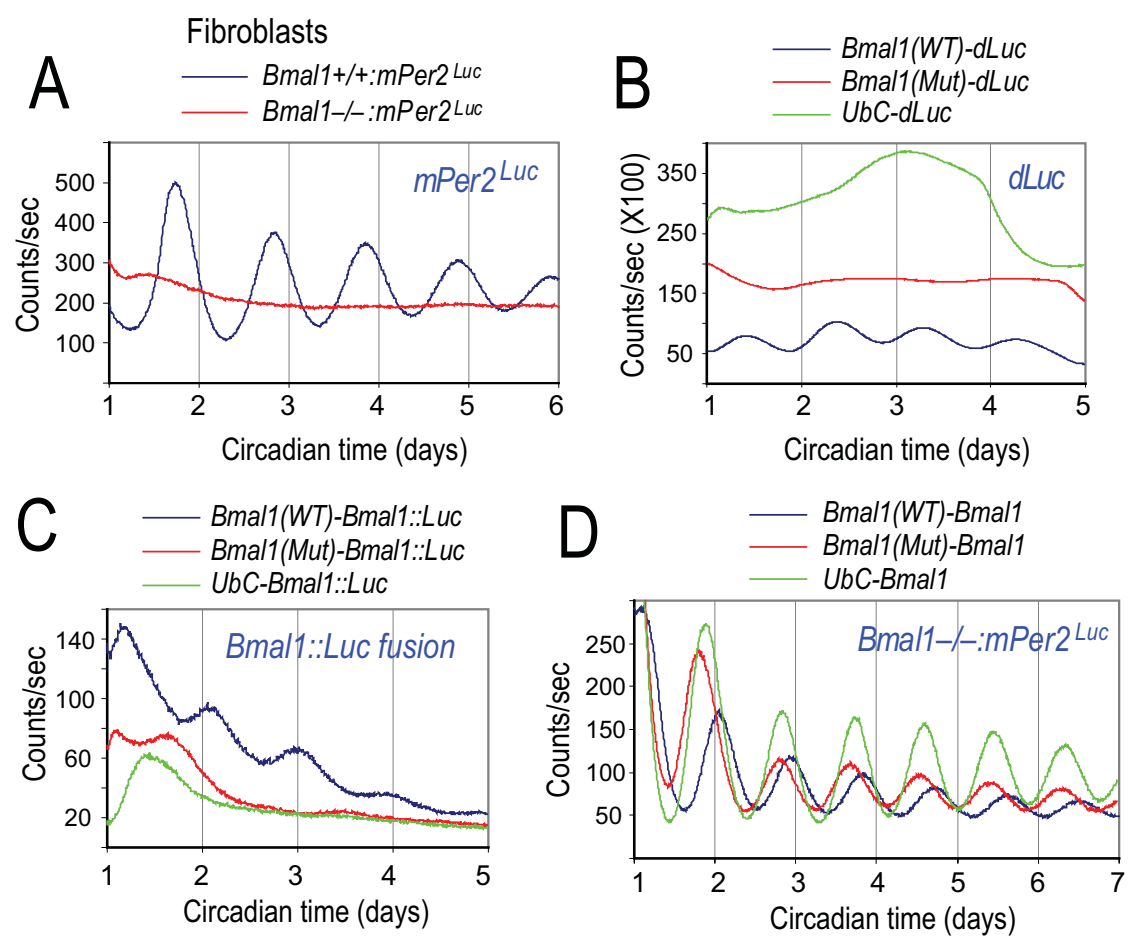

Figure 3. Cyclic expression of BMAL1 is not required for intracellular core clock function. (A) Bioluminescence patterns of fibroblasts

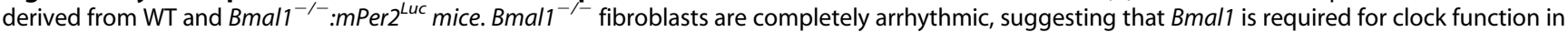
fibroblasts. Circadian time: days after explant medium change. (B) Bioluminescence patterns in wild-type fibroblasts transduced with a lentiviral $d L u c$ reporter. Bmal1(WT): Bmal1 promoter containing WT RORE sequence. Bmal1(Mut): Bmal1 promoter containing mutated RORE sequences. UbC: Ubiquitin C promoter. Unlike the Bmal1(WT), the Bmal1(Mut) and UbC promoters do not confer rhythmic luciferase expression. Circadian time: days after explant medium change. (C) Bioluminescence patterns in wild-type fibroblasts transduced with a lentiviral Bmal1::Luc fusion reporter. Unlike the Bmal1(WT), the Bmal1(Mut) and UbC promoters do not confer rhythmic BMAL1::LUC fusion protein expression. These results suggest that BMAL1 protein does not cycle and that only promoters that contain functional circadian elements confer rhythmic fusion protein expression. Circadian time: days after explant medium change. (D) Representative records of mPer2 ${ }^{\text {Luc }}$ rhythms in Bmal1 ${ }^{-/-}$fibroblasts restored through genetic complementation. Lentiviral expression vectors carrying Bmal1 CDNA under control of different promoters were introduced into Bmal1 ${ }^{-1-}: \mathrm{mPer}^{\mathrm{Luc}}$ fibroblasts. The three promoters gave rise to similar levels of BMAL1 protein expression as determined by Q-PCR and Western blotting (data not shown). Both cyclically and constitutively expressed BMAL1 restored circadian rhythmicity in Bmal1 ${ }^{-1-}$ fibroblasts, suggesting that the rhythm of BMAL1 protein is not required for basic core clock function. Circadian time: days after explant medium change.

doi:10.1371/journal.pgen.1000023.g003

\section{In Vivo Genetic Evidence for Cry1 and Rorc Regulation by REV-ERBs}

The Rorc gene has at least two E-boxes within the promoter region, and its circadian expression pattern is similar to Cry 1 in the liver. In vitro studies suggest that Rorc transcription is regulated by BMAL1/CLOCK [31]. To verify the in vitro results, we demonstrated that, similar to the expression patterns of other BMAL1/CLOCK-regulated clock components, the Rorc mRNA rhythm was abolished in the Bmal1 ${ }^{-1-}$ mouse liver, confirming that Rorc is regulated by the core loop (Figure 4A).

Interestingly, however, we observed that mRNA levels of Rorc as well as Cry 1 are clearly elevated rather than reduced in Bmal1 ${ }^{-1-}$ liver. This was surprising at first given that BMAL1 is a known activator of Cry 1 and Rorc expression. However, it should not be so surprising given the complexity of transcriptional circuitry of the clock. Similarly, higher Cry 1 mRNA levels were also reported previously in Bmal1 ${ }^{-\prime-}$, Clock ${ }^{m / m}$ and Clock $^{-1-}$ mice [32,33]. A recent in silico study showed that Cry 1 and Rorc genes contain two types of circadian regulatory elements, the E-box and the RORE [31]. In vitro and in vivo evidence also supports the presence of RORE sites within the Cry 1 gene $[5,30]$. In the absence of E-box regulation, factors acting through the RORE, namely the RORs and REV-ERBs, are likely to govern $\mathrm{Cry} 1$ and Rorc transcription.
In line with this notion, Clock mRNA is also higher in Bmal1 ${ }^{-/-}$ liver (Figure 4A), and Bmal1 mRNA is higher in Clock $^{-/-}$mouse liver [33].

A recent study proposed dual activator and repressor functions of BMAL1/CLOCK, in which its repressor function explains the elevated Cry 1 expression in the absence of Bmal1 [32]. However, that study did not take into consideration $C r y 1$ gene regulation through the ROREs. In both WT and Bmal1 ${ }^{-}-$mouse liver, there exists a strong inverse correlation between Rev-erb $\alpha$ and Cry 1/Rorc mRNA levels: when Rev-erb $\alpha$ is high, Cry 1/Rorc is low, and vice versa (Figure 4A). Similar expression patterns were also observed in fibroblasts (Figures $1 \mathrm{~B}$ and $5 \mathrm{~B}$ ) and in Rev-erb $\alpha^{-1-}$ mice [5], and suggested from in silico and in intro studies [30,31]. Thus, the elevated Rorc and Cry 1 expression in the absence of Bmal1 may be regulated primarily by the REV-ERBs rather than the repressor function of BMAL1. We therefore sought to experimentally demonstrate this notion. We hypothesized that over-expression of Rev-erb $\alpha$ in Bmal1 ${ }^{-/-}$cells would bring down the expression levels of Cry 1 and Rorc. Because Cry 1 and Rorc genes are regulated similarly but Rorc is not expressed in fibroblasts, we focused our analysis on the Cry 1 gene in this cell type. To test this idea, we introduced Rev-erb $\alpha$ into $\mathrm{Bmal1}^{-1-}$ cells by lentivirusmediated delivery and obtained a Bmal1 ${ }^{-1-}:$ Rev-erb $\alpha$-OX fibro- 


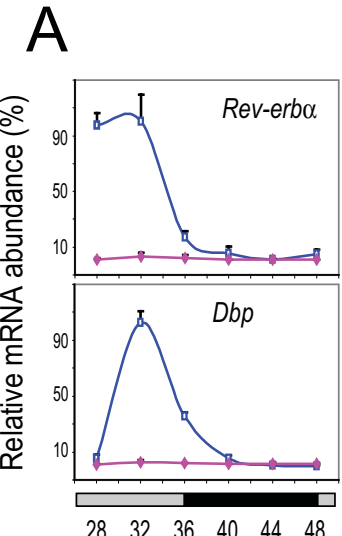

Liver $\rightarrow-B m a l 1+/+\longrightarrow B$ Bmal1-/-
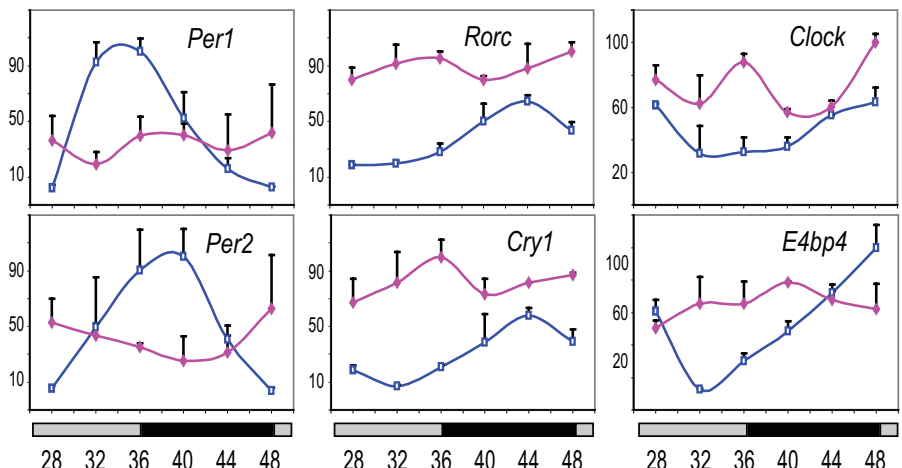

Circadian time (hours)
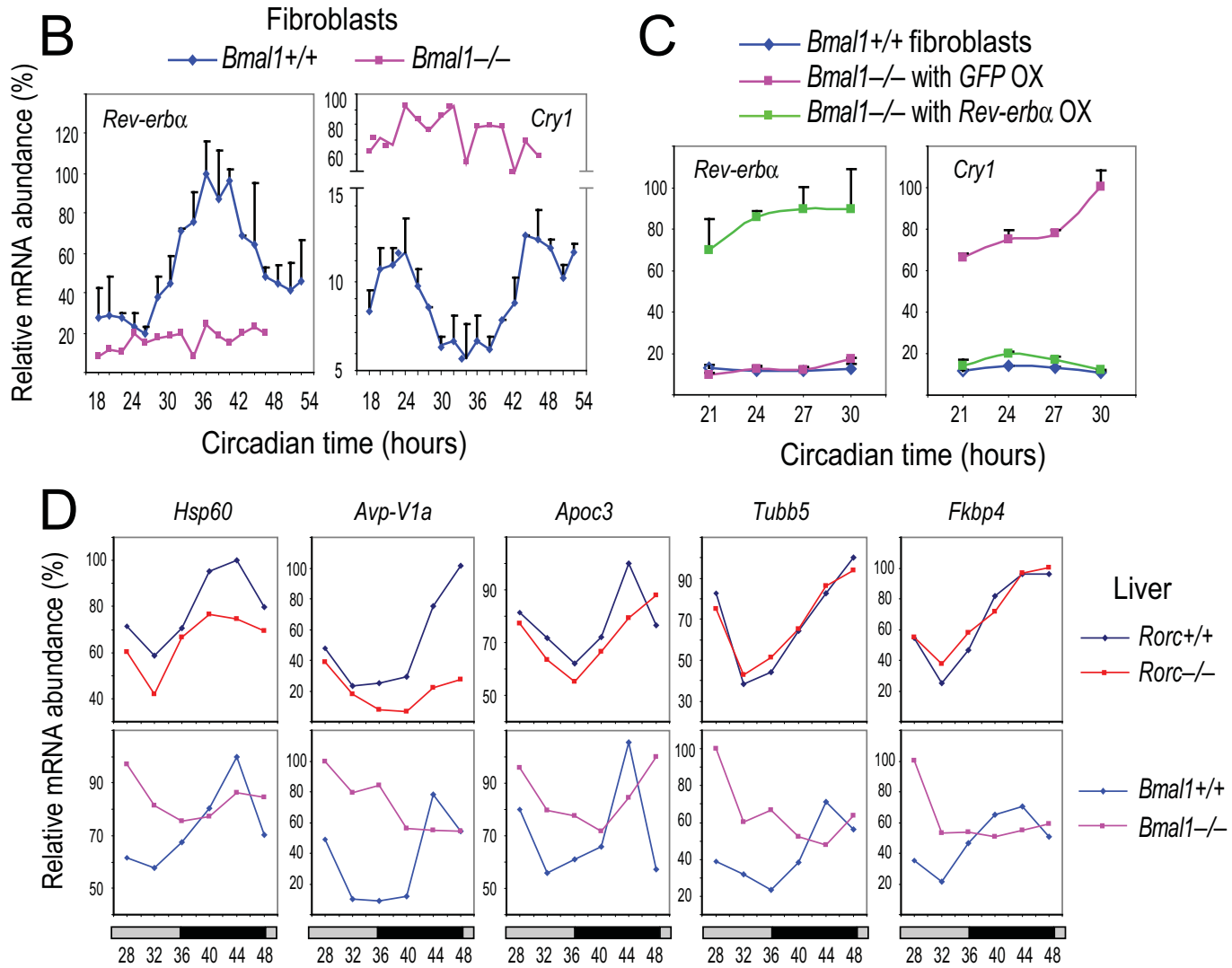

Circadian time (hours)

Figure 4. REV-ERBs play a prominent role in combinatorial regulation of Cry1 and Rorc. (A) Temporal mRNA expression profiles of clock genes in the liver of Bmal1 ${ }^{-1-}$ mice. Expression was analyzed at 4-hr intervals by Q-PCR. Values are expressed as percentage of maximum expression for each gene. Error bar represents standard deviation (SD) of expression levels from four mice. The clock genes are presented in four groups based on different mRNA expression patterns (phase and level) in WT and Bmal1 ${ }^{-/-}$mice. For instance, transcription of Cry1 and Rorc is elevated, rather than repressed, in the Bmal1 $1^{-1}$ liver. Circadian time: hours after animal release in constant darkness. (B) Temporal mRNA expression profiles of Rev-erb $\alpha$ and Cry 1 in Bmal1 ${ }^{-1-}$ fibroblasts. Expression was analyzed at 2-hr intervals by Q-PCR. Values are expressed as percentage of maximum expression for each gene. Results were confirmed in two independent time courses. Error bars represent SD of two culture samples for each cell line. Cry 1 mRNA levels are constantly high throughout the day and Rev-erb $\alpha$ expression is completely abolished in Bmal1 ${ }^{-/-}$fibroblasts, similar to results obtained from the liver. Circadian time: hours after serum treatment. (C) Over-expression (OX) of Rev-erb $\alpha$ represses elevated Cry 1 mRNA levels in Bmal1 ${ }^{-1-}$ fibroblasts. Expression of GFP and REV-ERB $\alpha$ is driven by a constitutive CAG promoter. Temporal mRNA expression was analyzed at 3-hr intervals by Q-PCR. Values are expressed as percentage of maximum expression for each gene. Results were confirmed in two independent experiments. Error bars represent SD of two culture samples for each cell line. REV-ERB $\alpha$ expression was confirmed by Q-PCR, and also by Western blotting (data not shown). Circadian time: hours after serum treatment. (D) Temporal mRNA expression profiles of clock-controlled output genes in the liver of Rorc ${ }^{-1-}$ and $B m a l 1^{-1-}$ mice. Experiments were performed as described in Figure 1A for Rorc ${ }^{-1-}$ mice and Figure $4 \mathrm{~A}$ for Bmal1 ${ }^{-1-}$ mice. As for Bmal1 and Cry 1, the prominent role of REV-ERBs in regulating transcription explains the elevated mRNA levels of these output genes in $B m a l 1^{-1-}$ mice. For clarity, error bars representing SD from four mice ( $<10 \%$ for each gene) were omitted. Circadian time: hours after animal release in constant darkness.

doi:10.1371/journal.pgen.1000023.g004 
A

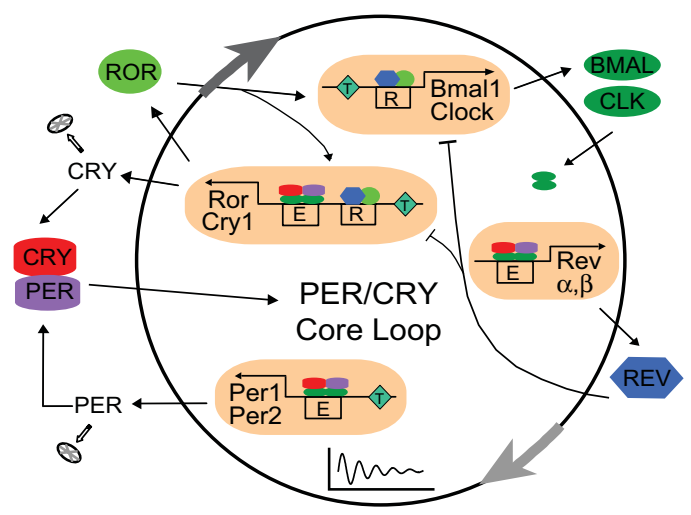

Bmal1
knockout $\left\{\begin{array}{l}\text { Genetic rescue by } \\ \text { cyclic or non-cyclic } \\ \text { BMAL1 expression }\end{array}\right.$

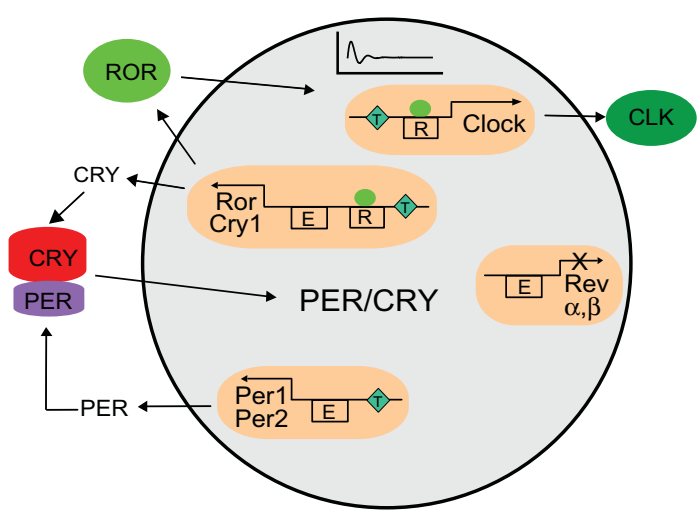

B

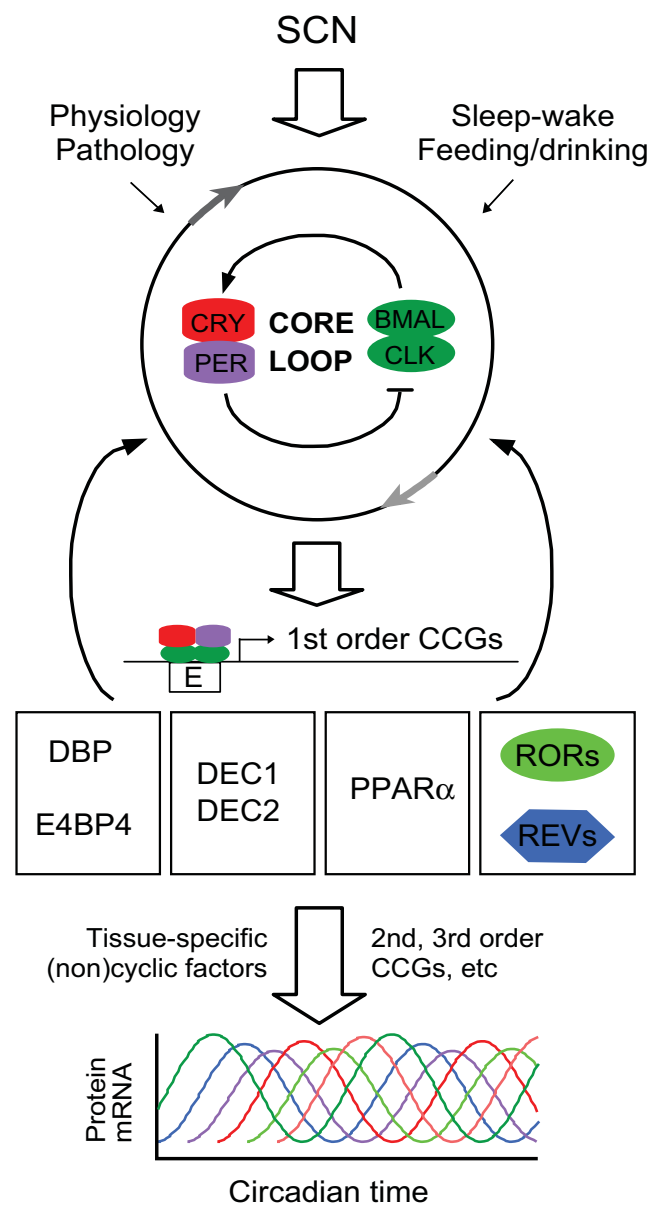

Figure 5. Model for circadian core clock mechanism and function. (A) Different transcriptional regulation gives rise to differential phasing of clock genes. PER/CRY and BMAL1/CLOCK (BMAL/CLK) form the core feedback loop mediated by the E-box. The RORs and REV-ERBs are directly regulated by the core loop and provide additional positive and negative feedbacks, respectively, to Bmal1/Clock transcription. Four main types of gene regulatory mechanisms exist in a wild-type cell (top): 1) Rev-erb $\alpha$ and $\beta$ (Rev) are driven primarily by E-box-mediated transcription, 2) Per1 and Per2 are regulated by BMAL1/CLOCK and additionally by a tonic signal input (T), 3) Cry1 and Rorc are regulated by BMAL1/CLOCK and ROR/REV as well as a tonic signal, and 4) Bmal1 and Clock are regulated by ROR/REV and a tonic signal. These different modes of transcriptional regulation provide the mechanistic basis for the different phases of their mRNAs (e.g. Rorc phase-delays Rev-erb $\alpha$ ) in WT cells and the differential levels of expression (e.g., diminished REV leads to Cry1 up-regulation) in Bmal1 ${ }^{-/-}$cells. BMAL1 is an essential clock component, and Bmal1 ${ }^{-/-}$cells are completely arrhythmic (bottom). However, its rhythmic patterns of mRNA and protein expression are not required for core clock function. Genetic complementation by either cyclically or constitutively expressed Bmal1 was able to restore circadian rhythmicity in $\mathrm{Bmal1}^{-/-}$cells. We suggest that the robustness of the core loop in the absence of rhythmic BMAL1 is retained by coordinated regulation of transcriptional and post-translational mechanisms, including particularly protein turnover and synchronous nuclear translocation of PER/CRY proteins despite the differential phases and/or lack of rhythmicity of their mRNAs (see discussion). In both the core loop and ROR/REV/Bmal1 loop, the repressors play more dominant roles than the activators. (B) The interlocking loops connect the core loop to temporal regulation of local output networks. Peripheral tissues are coordinated by the SCN in vivo, and the states of peripheral oscillators are also influenced by behavior, physiology, and pathology. The core loop directly controls expression of $1^{\text {st }}$ order CCGs, subsequently forming a cascade of rhythmic gene expression. The net result of this cascade is the appropriately timed production of proteins important for local physiology, which collectively contribute to coordinated circadian behavior and physiology at the organismal level. In this context, the interlocking loops, including the ROR/REV/Bmal1 loop and its constituents, are $1^{\text {st }}$ order CCGs and serve as important transmitters or integrators for local circadian biology.

doi:10.1371/journal.pgen.1000023.g005

blast cell line. Indeed, over-expressed REV-ERB $\alpha$ in $B m a l 1^{-1-}$ cells efficiently repressed Cry 1 mRNA to levels similar to those in WT cells (Figure 4G). Taken together, we provide direct in vivo genetic and molecular evidence to support the notion that Cry 1 and Rorc are regulated not only by BMAL1/CLOCK but also directly by the REV-ERBs (Figure $5 \mathrm{~A}$ ), which is the underlying molecular mechanism for elevated Cry 1 expression in Bmal1 $^{-1-}$ cells.

\section{Different Transcriptional Regulation Explains Differential Phasing of Clock Genes}

Interestingly, the mRNA levels of other clock genes in the liver of Bmal1 $^{-1-}$ mice are also very different (Figure 4A): Dbp and Rev-erb $\alpha$ expression is dramatically reduced, and Per 1 and Per 2 are expressed at constant intermediate levels, consistent with sustained $m P e r 2^{L u c}$ expression in Bmal1 ${ }^{-1-}$ cells (Figure 3A), whereas Rorc, Cry 1, Clock and E4bp4 are clearly de-repressed. Based on mRNA 
expression patterns in both WT and Bmal1 ${ }^{-/-}$cells (Figure 4A), we suggest the following transcriptional regulatory scheme for clock gene expression (Figure 5A): Dbp and Rev-erb $\alpha$ are activated primarily by BMAL1/CLOCK via the E-boxes, and that this Ebox-mediated circadian regulation is essentially eliminated in the absence of BMAL1 (and thus PER/CRY-mediated repression via the E-box is no longer relevant). Per1 and Per2 are activated by BMAL1/CLOGK and other non-circadian mechanisms, accounting for the intermediate mRNA levels of Per1 and Per2 in Bmal1 ${ }^{-1-}$ mice. Rorc and Cry 1 are regulated not only by BMAL1/CLOCK but also by RORs/REV-ERBs via the RORE. Bmal1, Clock and E4bp4 are regulated by RORs/REV-ERBs via the RORE.

The different regulatory mechanisms offer mechanistic explanations for distinct phases of clock gene expression rhythms observed in vivo (Figure 5A). Dbp is controlled by BMAL1/ CLOCK via the E-box, while E4bp4 is primarily regulated via RORE, explaining why the E4bp4 rhythm is in phase with Bmal1 and Clock, but is antiphasic to Dbp. Rev-erb $\alpha$ and Rorc are both activated by BMAL1/CLOCK, but Rorc is also repressed by REVERBs, explaining how Rorc mRNA accumulation is phase-delayed compared to that of Rev-erb $\alpha$. Additional regulation of Per 1 and Per2 by non-circadian factors (and possibly also by E4bp4) may cause a phase-delay compared to $D b p$ and Rev-erb $\alpha$. In summary, our data provides novel mechanistic insights into how the genes in the clock circuitry are regulated in vivo [31].

\section{RORc and REV-ERBs Control Rhythmic Expression of Output Genes}

The RORs appear to regulate the amplitude of target gene expression, while the REV-ERBs regulate the rhythmic expression of Bmall and also participate in combinatorial regulation of Cry 1 . As these regulatory mechanisms are not required for basic clock function, we suggest that the ROR/REV/Bmal1 loop and its constituents provide additional opportunities to control timespecific expression of output genes in local clock physiology, especially in peripheral tissues. In this context, the differential tissue expression patterns of the RORs also provide additional opportunities for tissue-specific local circadian biology (Figure 5B).

The combinatorial regulatory mechanism provides a novel strategy for identifying and validating target genes of the RORs and REV-ERBs, as well as differentiating RORE-containing genes from those containing both RORE and E-boxes (Figure 4A). Here we examined several of the genes that exhibit phases similar to Bmal1 or Cry 1 in the liver and contain potential RORE sequences $[25,26]$. For example, mRNAs of heat-shock protein 60 (Hsp60), arginine vasopressin receptor 1A $(A v p-V 1 a)$ and Apoc3 were reduced in the liver of orc $^{-1-}$ mice, especially at peak time (CT40-48), reflecting reduction of RORE-mediated activation, but their mRNA levels were up-regulated in Bmal1 $^{-1-}$ mice at CT28-36, corresponding to the trough time in WT, reflecting loss of E-box-mediated REV-ERB expression with subsequent relief of RORE-mediated repression. Tubulin beta 5 (Tubb5) and peptidylprolyl cis-trans isomerase FK506 binding protein 4 (Fkbp4) also exhibited significantly higher mRNA levels at CT28-36 in Bmal1 $^{-/-}$mice, but their expression levels were not affected in Rorc $^{-1-}$ mice. Thus, cyclic RORE-mediated activation and/or repression may modulate expression patterns of specific target genes involved in important biological processes in a tissue-specific manner.

\section{Discussion}

In summary, our results suggest that the intracellular core clock loop is intrinsically resilient and is largely responsible for generating and maintaining basic circadian rhythmicity. The multiple additional interlocking loops contribute to, but are not necessary for, core clock function. Cellular rhythms are intrinsically stochastic. However, intercellular coupling mechanisms uniquely present in the SCN play a dominant role in maintaining the robustness of the SCN and the body timekeeping system $[2,23]$. We therefore suggest that interlocking loops function mainly to provide additional regulatory mechanisms to modulate the phases of gene expression locally.

\section{Regulation of the Bmal1 Interlocking Loop}

Previous studies using mice deficient in Rora, Rorb or Rev-erb $\alpha$ function strongly suggested functional redundancy among the ROR and REV-ERB family members [5,6,10,11]. Mutation of Rora was shown to reduce Bmall mRNA amplitude both in the SCN [6] and in fibroblasts [11], and Rev-erb $\alpha$ deletion resulted in much higher levels of Bmal1 transcription [5], but Bmal1 rhythms were still retained despite either deficiency.

While null mutations in core clock genes typically lead to severe impairment of clock function (see below), deficiencies in clock components within the ROR/REV/Bmal1 loop only produce modest clock phenotypes [5-7,10,11]. The ROR/REV/Bmal1 loop is thus thought to provide a "stabilizing" function. However, mice deficient in core clock components (e.g., $\mathrm{Per} 1^{-/-}, \mathrm{Per} 2^{-/-}$or $\mathrm{Clock}^{\mathrm{m} / \mathrm{m}}$ mice) also similarly show less precise or less persistent circadian rhythms [19-22].

In this study, we investigated the redundancy of functions among the ROR and REV-ERB family members and clarified their roles in regulating Bmal1 expression. To circumvent pleiotropic effects of gene deletion, we directly tested this "stabilization function" hypothesis in cell-autonomous clock models by perturbing the BMALl rhythm. We demonstrated that cells with Rev-erb $\alpha$-knockout or Rev-erbb-knockdown still rhythmically express Bmal1. The Bmal1-dLuc rhythm could be abolished only when both Rev-erb $\alpha$ and $b$ were disrupted (Figure 2B). Thus, REV-ERB $\alpha$ and REV-ERB $\beta$ are required for Bmal1 rhythmicity, and they are functionally redundant. In contrast, the RORs are not required for Bmal1 rhythmicity (Figure 1). Thus, the REVERBs play a more prominent role than the RORs in regulating the rhythmic expression of Bmal1.

\section{The Robustness of the Circadian Clock}

The current models for mouse and fly circadian clocks indicate that the process of evolution has produced a genetic circuitry substantially more complex than a simple transcriptional feedback scheme. Presumably, robustness is a key feature of circadian control that is likely to be under selective pressure, as it would underlie the adaptive significance of a particular physiological rhythm. Robustness is the ability of a system to maintain essential properties despite internal noise and external perturbations, a property which is prevalent in biological control circuits [34]. From a circadian clock perspective, the key measures of robustness are precision (period stability over time), persistence (how long a given clock system sustains rhythm amplitude without a resetting signal), and accuracy (period consistency of cells, tissues, or organisms). It should be noted, however, that period variation and alteration may be an indicator of robustness, not necessarily instability. Mechanisms contributing to the robustness of the clock system include additional interlocking loops, gene redundancy, maintenance of amplitude, and intercellular coupling.

In contrast to the proposed "stabilizing" role of the ROR/ REV/Bmal1 loop, we found that Per2-dLuc expression is rhythmic even in cells deficient in both REV-ERB $\alpha$ and $\beta$ function (Figure 2D) or expressing constitutive BMALl protein (Figure 3D). 
This provides unambiguous evidence from cell-autonomous preparations that Bmal1 mRNA and protein rhythms are not essential for the basic operation of the intracellular clock. In accord with our findings, constitutively expressed Bmal1 in the SCN of Bmal1 ${ }^{-/-}$mice was able to rescue circadian behavioral rhythmicity [24].

Using real-time bioluminescence imaging to monitor Per2 gene expression in tissues and cells from mutant mice [23], we recently found that both Per1 and Per2 are required for sustained cellautonomous rhythms in individual cells. Importantly, intercellular coupling in the SCN can compensate for clock gene deficiency, preserving sustained cellular rhythmicity in mutant SCN slices and behavior. Thus, SCN intercellular coupling is essential not only to synchronize component cellular oscillators but also for robustness against genetic perturbations. In this context, it is reasonable to presume that, owing to intercellular coupling, an SCN ensemble that expresses non-cyclic Bmal1 mRNA/proteins would still exhibit robust Per2 and Cry 1 rhythms. However, Rora ${ }^{s g / s g}$, Rorb $^{-1-}$ and Rev-erb $\alpha^{-/-}$mice exhibit circadian period defects in behavior, albeit very mild. Thus, to address the cellular basis of circadian behavior, future studies using real-time bioluminescence technology are needed to examine the molecular dynamics of circadian rhythmicity in the SCN ensemble as well as in dissociated SCN neurons of single and double loss-of-function mutants of the Ror and Rev-erb genes.

However, the nonessential ROR/REV/Bmal1 loop in the basic intracellular clock mechanism clearly regulates expression rhythm and amplitude of many output genes. Maintaining a biologically relevant high-amplitude rhythm of gene expression also contributes to the robustness of the clock system. The significance of amplitude in clock function is supported by a recent study showing that $C l o c k^{m / m}$ mice exhibited increased efficacy in response to resetting stimuli due to reduced circadian amplitude in the SCN pacemaker [35]. Similarly, the ROR/REV/Bmal1 loop may also benefit organismal survival in the natural environment by contributing to robust high-amplitude rhythms [13]. Furthermore, this interlocking loop may contribute to transduction of environmental cues to the core loop [13]. In line with this notion, behavioral studies have implicated Rev-erb $\alpha$ and Rorb in photic responses $[5,36]$. It is interesting to note that there appears to be a delayed phase of Per2 oscillation in cells that express arrhythmic Bmal1 mRNA and protein (Figures 3D and 4D). As Per2 induction may be involved in synchronization [37], it is possible that the $\mathrm{ROR} / \mathrm{REV} /$ Bmal1 loop plays an important role in circadian entrainment of peripheral oscillators.

\section{Potential Tonic Signaling Input to Circadian Intracellular Transcriptional Networks}

The resilience of the intracellular core clock function without inputs from the ROR/REV/Bmal1 loop indicates that general cellular mechanisms must play important roles in attaining robust clock function, including particularly post-translational modifications and protein turnover affecting subcellular translocation and activities of clock components. In particular, results from this study strongly suggested the involvement of tonic signaling in clock function (Figure 5A). In Bmal1 ${ }^{-1-}$ cells, transcription of Rev-erb $\alpha$ and $b$ is completely abolished, whereas Per1 and Per2 maintain intermediate transcription levels throughout the day. Any contribution from $D b p / E 4 b p 4$ is minimal in these cells, as the level of the DBP activator is too low and the E4BP4 repressor is constantly high (Figure 4A). Rather, it is likely that, without BMAL1/CLOCK activators, Per1 and Per2 transcription is maintained through a non-circadian, tonic signal input such as the cyclic AMP response element-binding (CREB) signal trans- duction cascade. Similarly, presence of tonic signaling and lack of repression by the REV-ERBs are the primary cause for the constantly high levels of Cry 1, Rorc and Clock expression in Bmal1 ${ }^{-1-}$ cells. It is conceivable that the activating tonic signal input also explains why the ROR activators are dispensable for driving rhythmic transcription of Bmal1 provided that the REV-ERBs are present in the cells. It is likely that the balance between positive and negative regulators as well as tonic signaling determines clock gene expression at any given circadian time. The tonic signal input is usually overlooked in the WT genetic background, but is uncovered when the functions of positive and/or negative regulators are blocked (Figure 5A). Tonic signaling is also important to consider in interpreting effects of Per or Cry mutations on cellular rhythms in the SCI [2,23].

\section{Interlocking Loops Function Mainly To Regulate Circadian Outputs}

In addition to the ROR/REV/Bmall loop, other known interlocking loops or components include $D b p / E 4 b p 4$, Ppar $\alpha$, and Dec1/Dec2 (Figure 5B). These secondary loops are directly regulated by the core loop through the E-boxes [31]. E4BP4 and DBP, analogs of dVRI and dPDP1 in flies [16], form an oscillatory loop by feeding back to regulate Per2 transcription [31,38-40]. DEC1 and DEC2 form another feedback loop, functioning to repress E-box-mediated transcription [41]. Very recently, clockwork orange (cwo), a Dec homolog, has been identified in Drosophila and shown to regulate rhythm amplitude [42-44]. The PPAR $\alpha$ loop, on the other hand, feeds back to activate Bmall expression through potential PPAR response elements in the Bmal1 gene [45-47]. Interestingly, peroxisome proliferator-activated receptor-gamma coactivator 1 alpha $(\mathrm{PGC}-1 \alpha)$ has recently also been shown to activate Bmall expression by acting as a ROR activator [48].

Unlike the requirement for core clock components-PER/CRY [23], CLOCK [49], and BMAL1 (Figure 3A in this study), none of the interlocking loops discussed above appears to be required for basic clock function (in this study) [47,48,50-52]. In addition, the $\mathrm{ROR} / \mathrm{REV} /$ Bmal1 loop function is not conserved between mammals and flies $[7,16]$. Rather, it's conceivable that the major function of the interlocking loops is to transmit circadian signals to control local output genes at different times during the day, as required for circadian behavior and physiology. Direct transduction of circadian information to local output rhythms can be more efficiently accomplished through first-order clock-controlled genes $\left(1^{\text {st }}\right.$ order CGGs) that are directly regulated by the core loop, which subsequently regulate expression of $2^{\text {nd }}$ and $3^{\text {rd }}$ order CCGs (Figure 5B). In this context, the interlocking loops and their constituents serve as the $1^{\text {st }}$ order CCGs. Most CGGs exhibit tissue-specific expression patterns, and many are involved in ratelimiting steps of reactions important for the main functions of their respective tissues $[3,26,53,54]$. The core loop components are well conserved among various tissues, while the $1^{\text {st }}$ order CGGs such as the RORs may be highly tissue-specific. The $1^{\text {st }}$ order CGGs could also establish crosstalk with other tissue-specific circadian or noncircadian factors. Thus, the multiple interlocking loops provide an efficient means not only to amplify circadian signals but also to provide additional phase information for local outputs (Figure 5B). For instance, the antiphasic expression of $D b p$ and E4bp4 is known to regulate the rhythmic production of many proteins involved in bile acid production, drug metabolism, and xenobiotic detoxification in liver and kidney [3,38,55].

The RORs and REV-ERBs are known to be involved in many cellular, physiological, and pathological processes [4,56-60]. For example, RORa and RORc regulate phase I and II metabolism 
[61]. RORa activates and REV-ERB $\alpha$ represses genes encoding apolipoprotein C-III (ApoC-III) and ApoA1, key proteins in plasma triglyceride and lipoprotein metabolism [60]. RORa and REV-ERB $\beta$ also regulate many genes involved in lipid homeostasis in skeletal muscle cells $[62,63]$. REV-ERB $\alpha$ was shown to regulate circadian expression of plasminogen activator inhibitor type 1 (PAI-1), suggesting a role in thrombosis [64]. Interestingly, crosstalk exists between REV-ERB $\alpha$ and PPAR nuclear receptors, which are important factors regulating lipid and glucose homeostasis and inflammation [60]. Recently, anatomical expression profiling of nuclear receptors has revealed significant metabolic implications of peripheral clock biology $[27,28,65]$.

With the interlocking loops as entry points, future studies should focus on more detailed characterization of the transcriptional circuitry regulating time- and tissue-specific outputs involved in circadian behavior, physiology, and pathology. Knowledge of circadian signaling and clock-regulated local biology will likely have important implications for the pathogenesis and treatment of diseases such as metabolic syndrome, heart disease, diabetes, and obesity.

\section{Materials and Methods}

\section{Animal}

Bmal1 $^{-/-}$mouse line was obtained from Chris Bradfield at the University of Wisconsin, Rorc ${ }^{-1-}$ line from Dan Littman at New York University, and $m P e r 2^{L u c}$ transgenic reporter line from Joe Takahashi at Northwestern University. Knockout mice were bred with $m P e r 2^{L u c}$ reporter mice to obtain homozygous knockouts harboring the mPer2 $2^{L u c}$ reporter. Wheel-running assays were performed and analyzed as described previously [23]. Behavioral phenotypes of these mice were similar to the respective knockout animals not carrying the reporter. All animal studies were conducted in accordance with the regulations of the Committees on Animal Care and Use at The Scripps Research Institute.

\section{Cell Culture, Transfection, and Lentiviral Production and Infection}

Explants of SCN and peripheral tissues were dissected and cultured as previously described [23,66]. Primary mouse fibroblasts were generated from tails by a standard enzymatic digestion procedure [67]. Fibroblasts that spontaneously overcame replicative senescence (immortalization) were used in this study. All fibroblasts were cultured in DMEM supplemented with $10 \%$ fetal bovine serum and antibiotics, and grown to confluence prior to bioluminescence recording or harvesting for mRNA time courses. MMH-D3 hepatocytes were cultured as described previously [68].

Recombinant lentiviruses were produced by transient transfection in 293T cells using the calcium-phosphate method as previously described [23]. Infectious lentiviruses were harvested at $48 \mathrm{hr}$ post-transfection and used to infect various cells. Cells infected with pLL3.7(GW)-shRNA constructs were sorted by FACS for the highest (10\%) GFP-expressing cells. Cells infected with pLV156-Per2-dLuc reporter [23] were sorted by FACS for GFP expression as described therein. Cells infected with pLenti6B4B2 constructs expressing proteins including Per2-dLuc and Bmal1-dLuc reporters were selected with $10 \mu \mathrm{g} / \mathrm{ml}$ Blasticidin and further propagated for further study.

\section{DNA and shRNA Constructs}

For cDNA expression constructs, DNA sequences including GFP, Bmal1, Rev-erb $\alpha$, Rev-erbb, the firefly Luciferase gene $(L u c)$, the rapidly degradable Luciferase gene $(d L u c)$, and Bmal1::Luc, were first cloned into pENTR/D-TOPO entry vector (Invitrogen). All promoter sequences including Bmal1(WT), Bmal1(Mut), UbC, Per2, and the composite $C A G$ promoter were first cloned into pENTR5'-TOPO vector (Invitrogen). The pENTR/D-TOPO-cDNA and pENTR-5' -promoter plasmid DNAs were then recombined with pLenti6/R4R2/V5-DEST destination vector (Invitrogen) in a MultiSite Gateway recombination reaction to generate expression constructs (see Text S1).

For shRNA expression constructs, we first designed and generated nine 29-bp long oligo-nucleotides against different regions of the Rev-erbb gene. Synthetic oligonucleotides were annealed and cloned into pENTR/U6 (Invitrogen) according to manufacture's instruction and subsequently cloned into the pLL3.7GW vector which harbors a CMV-driven GFP gene [69]. Among the tested nine shRNA constructs against Rev-erbb, three (designated $\beta 1, \beta 2$ and $\beta 3$ ) were found that were nonoverlapping and efficiently depleted over-expressed REV-ERB $\beta$ protein in transfected HEK293T cells as tested by Western blot analysis (data not shown) and knocked down Rev-erbb mRNA expression in fibroblasts as tested by Q-PGR. The parental pLL3.7GW empty vector and a nonspecific shRNA construct were used as controls (see Text S1).

\section{Tissue Harvest and Quantitative PCR (Q-PCR)}

For liver and lung, mice were first entrained to regular lightdark cycles and then released to constant darkness, and peripheral tissue samples were harvested $28 \mathrm{hr}$ later. Total RNAs from liver and lung were first prepared using Trizol reagents (Invitrogen) followed by further purification using RNeasy mini kit (Qiagen). For fibroblasts, cell growth, serum shock were performed as previously described [5-7,10,23]. Total RNAs from fibroblasts were prepared using RNeasy mini kit.

Total RNAs were transcribed to cDNA using $1^{\text {st }}$ strand SuperScript III reverse transcriptase (Invitrogen). Q-PCR was performed using an iCycler thermal cycler with the MyiQ optical module (BioRad) as described previously [6,23]. Transcript levels for each gene were normalized to Gapdh. Average relative expression ratios for each gene were expressed as a percentage of the maximum ratio at peak expression (see Text $\mathrm{S} 1$ ).

\section{Bioluminescence Recording and Data Analysis}

Bioluminescence patterns were monitored using a LumiCycle luminometer (Actimetrics) as previously described [23]. Briefly, after change to fresh explant medium at ambient temperature, culture dishes containing cells or explants were sealed and placed into the luminometer, which was kept inside a standard tissue culture incubator at $36^{\circ} \mathrm{C}$. Bioluminescence from each dish was continuously recorded with a photomultiplier tube (PMT) for $\sim 70 \mathrm{sec}$ at intervals of 10 minutes. Raw data (counts/sec) were plotted against time (days) in culture. For analysis of rhythm parameters, we used the LumiCycle Analysis program (Actimetrics). Raw data were baseline fitted, and the baselinesubtracted data were fitted to a sine wave (damped), from which the period was determined. For samples that showed persistent rhythms, goodness-of-fit of $>80 \%$ was usually achieved. Due to high transient luminescence upon medium change, the first cycle was usually excluded from rhythm analysis. For FFT spectral analysis (RelAmp) of Bmal1-dLuc oscillations, LumiCycle Analysis version 2.10 was used, in which polynomial order was set at 3 for background subtraction, the first cycle of data was usually excluded, Blackman-Harris windowing was checked (power spectrum unchecked), and circadian range was defined at 20$30 \mathrm{hr}$. 


\section{Supporting Information}

Figure S1 ROR/REV-ERB expression patterns and Rorc-/animal behavioral rhythms. (A) Tissue-specific expression of the Ror- and Rev-erb genes. Total RNA was isolated from the tissues indicated, and gene expression was determined by standard reverse transcription and PCR (RT-PCR) followed by agarose gel electrophoresis. (B) Temporal mRNA expression profiles of Bmal1, Dbp, Rora, and Rorc in wild-type fibroblasts and hepatocytes. Expression was analyzed at 4-hr intervals by quantitative PCR (QPCR). Values are expressed as percentage of maximum expression for each gene. Error bars represent standard deviation (SD) of expression levels from two culture samples. Circadian time: hours after serum treatment. (C) Summary of Rora, Rorb, and Rorc expression in the SCN, liver, and fibroblasts. Curved line: rhythmic expression. Flat line: arrhythmic expression. NE: not expressed or expression not detected. Note that in fibroblasts, Rorb and Rorc are not detected, and Rora expression does not display a distinct mRNA rhythm. Rorc is not expressed in the SCN, but is rhythmically expressed in liver. (D) Double-plot actograms for $B c l-x L T g$ controls and homozygous Rorc-/-:Bcl-xLTg mice. Rorc-/- mice displayed normal circadian locomotor activity under constant darkness and normal phase shifts in response to

\section{References}

1. Young MW, Kay SA (2001) Time zones: A comparative genetics of circadian clocks. Nat Rev Genet 2: 702-715.

2. Liu AC, Lewis WG, Kay SA (2007) Mammalian circadian signaling networks and therapeutic targets. Nat Chem Biol 3: 631-639.

3. Reppert SM, Weaver DR (2002) Coordination of circadian timing in mammals. Nature 418: 935-941.

4. Giguere V (1999) Orphan nuclear receptors: From gene to function. Endocrine Rev 20: 689-725.

5. Preitner N, Damiola F, Molina LL, Zakany J, Duboule D, et al. (2002) The orphan nuclear receptor REV-ERB alpha controls circadian transcription within the positive limb of the mammalian circadian oscillator. Cell 110 : 251-260.

6. Sato TK, Panda S, Miraglia LJ, Reyes TM, Rudic RD, et al. (2004) A functional genomics strategy reveals Rora as a component of the mammalian circadian clock. Neuron 43: 527-537.

7. Emery P, Reppert SM (2004) A rhythmic ror. Neuron 43: 443-446.

8. Triqueneaux G, Thenot S, Kakizawa T, Antoch MP, Safi R, et al. (2004) The orphan receptor Rev-erbalpha gene is a target of the circadian clock pacemaker. J Mol Endocrinol 33: 585-608.

9. Guillaumond F, Dardente H, Giguere V, Cermakian N (2005) Differential control of Bmall circadian transcription by REV-ERB and ROR nuclear receptors. J Biol Rhythms 20: 391-403.

10. Andre E, Conquet F, Steinmayr M, Stratton SC, Porciatti V, et al. (1998) Disruption of retinoid-related orphan receptor beta changes circadian behavior, causes retinal degeneration and leads to vacillans phenotype in mice. EMBO J 17: 3867-3877.

11. Akashi M, Takumi $\mathrm{T}$ (2005) The orphan receptor ROR alpha regulates circadian transcription of the mammalian core-clock Bmal1. Nat Struct Mol Biol 12: $441-448$.

12. Gyran SA, Buchsbaum AM, Reddy KL, Lin MC, Glossop NRJ, et al. (2003) vrille, Pdp1, and dClock form a second feedback loop in the Drosophila circadian clock. Cell 112: 329-341.

13. Allada R (2003) Circadian clocks: A tale of two feedback loops. Cell 112: 284-286.

14. Yang Z, Sehgal A (2001) Role of molecular oscillations in generating behavioral rhythms in Drosophila. Neuron 29: 453-467.

15. Kim EY, Bae K, Kim DY, Ng FS, Glossop NRJ, et al. (2002) Drosophila CLOCK protein is under posttranslational control and influences light-induced activity. Neuron 34: 69-81.

16. Hardin PE (2005) The circadian timekeeping system of Drosophila. Curr Biol 15: R714-R722

17. Steinmayr M, Andre E, Conquet F, Rondi-Reig L, Delhaye-Bouchaud N, et al. (1998) Staggerer phenotype in retinoid-related orphan receptor alpha-deficient mice. Proc Natl Acad Sci U S A 95: 3960-3965.

18. Hamilton BA, Frankel WN, Kerrebrock AW, Hawkins TL, FitzHugh W, et al. (1996) Disruption of the nuclear hormone receptor ROR alpha in staggerer mice. Nature 379: 736-739.

19. Zheng BH, Albrecht U, Kaasik K, Sage M, Lu WQ, et al. (2001) Nonredundant roles of the mPerl and mPer2 genes in the mammalian circadian clock. Cell 105: 683-694. a light pulse, compared to controls. Yellow shading represents the light period of LD cycles. Red arrows indicate a light pulse applied at CT16.

Found at: doi:10.1371/journal.pgen.1000023.s001 (1.26 MB PDF)

Text S1 Supplemental Materials and Methods. (A) Expression vector construction. (B) shRNA vector construction. (C) TaqMan PCR primers and probes used in this study.

Found at: doi:10.1371/journal.pgen.1000023.s002 (0.10 MB PDF)

\section{Acknowledgments}

We thank Dan Littman for Rorc ${ }^{-1-}$ mice, Chris Bradfield for Bmal1 ${ }^{-/-}$ mice, Bruce Hamilton for Rora ${ }^{\mathrm{sg} / \mathrm{sg}}$ mice, and Ueli Schibler for Rev-erb $\alpha^{-/-}$ mouse tail biopsies. We thank Oded Singer for help in siRNA design. We thank Warren Lewis and Tsuyoshi Hirota for critical reading of the manuscript. We also thank Trey Sato, Satchin Panda, and John Hogenesch for discussions during early phases of this project.

\section{Author Contributions}

Conceived and designed the experiments: AL SK. Performed the experiments: AL HT EZ AP DW. Analyzed the data: AL. Contributed reagents/materials/analysis tools: AL. Wrote the paper: AL DW SK.

20. Bae K, Jin XW, Maywood ES, Hastings MH, Reppert SM, et al. (2001) Differential functions of mPer1, mPer2, and mPer3 in the SCN circadian clock. Neuron 30: 525-536.

21. Cermakian N, Monaco L, Pando MP, Dierich A, Sassone-Corsi P (2001) Altered behavioral rhythms and clock gene expression in mice with a targeted mutation in the Periodl gene. EMBO J 20: 3967-3974.

22. Vitaterna MH, King DP, Chang AM, Kornhauser JM, Lowrey PL, et al. (1994) Mutagenesis and mapping of a mouse gene, Clock, essential for circadian behavior. Science 264: 719-725.

23. Liu AC, Welsh DK, Ko CH, Tran HG, Zhang EE, et al. (2007) Intercellular coupling confers robustness against mutations in the SCN circadian clock network. Cell 129: 605-616.

24. McDearmon EL, Patel KN, Ko CH, Walisser JA, Schook AC, et al. (2006) Dissecting the functions of the mammalian clock protein BMALl by tissuespecific rescue in mice. Science 314: 1304-1308.

25. Ueda HR, Chen WB, Adachi A, Wakamatsu H, Hayashi S, et al. (2002) A transcription factor response element for gene expression during circadian night. Nature 418: 534-539.

26. Panda S, Antoch MP, Miller BH, Su AI, Schook AB, et al. (2002) Coordinated transcription of key pathways in the mouse by the circadian clock. Cell 109: 307-320.

27. Yang X, Downes M, Yu RT, Bookout AL, He W, et al. (2006) Nuclear receptor expression links the circadian clock to metabolism. Cell 126: 801-810.

28. Bookout AL, Jeong Y, Downes M, Yu RT, Evans RM, et al. (2006) Anatomical profiling of nuclear receptor expression reveals a hierarchical transcriptional network. Cell 126: 789-799.

29. Sun ZM, Unutmaz D, Zou YR, Sunshine MJ, Pierani A, et al. (2000) Requirement for ROR gamma in thymocyte survival and lymphoid organ development. Science 288: 2369-2373.

30. Etchegaray JP, Lee C, Wade PA, Reppert SM (2003) Rhythmic histone acetylation underlies transcription in the mammalian circadian clock. Nature 421: $177-182$.

31. Ueda HR, Hayashi S, Chen WB, Sano M, Machida M, et al. (2005) Systemlevel identification of transcriptional circuits underlying mammalian circadian clocks. Nat Genet 37: 187-192.

32. Kondratov RV, Shamanna RK, Kondratova AA, Gorbacheva VY, Antoch MP (2006) Dual role of the CLOCK/BMAL1 circadian complex in transcriptional regulation. FASEB J 20: 530-532.

33. DeBruyne JP, Noton E, Lambert GM, Maywood ES, Weaver DR, et al. (2006) A clock shock: mouse CLOCK is not required for circadian oscillator function. Neuron 50: 465-477.

34. Stelling J, Sauer U, Szallasi Z, Doyle FJ, Doyle J (2004) Robustness of cellular functions. Cell 118: 675-685.

35. Vitaterna MH, Ko CH, Chang AM, Buhr ED, Fruechte EM, et al. (2006) The mouse Clock mutation reduces circadian pacemaker amplitude and enhances efficacy of resetting stimuli and phase-response curve amplitude. Proc Natl Acad Sci U S A 103: 9327-9332.

36. Masana MI, Sumaya IC, Becker-Andre M, Dubocovich ML (2007) Behavioral characterization and modulation of circadian rhythms by light and melatonin in C3H/HeN mice homozygous for the RORbeta knockout. Am J Physiol Regul Integr Comp Physiol 292: R2357-R2367. 
37. Kornmann B, Schaad O, Bujard H, Takahashi JS, Schibler U (2007) Systemdriven and oscillator-dependent circadian transcription in mice with a conditionally active liver clock. PLoS Biology 5: e34. doi:10.1371/journal. pbio.0050034.

38. Mitsui S, Yamaguchi S, Matsuo T, Ishida Y, Okamura H (2001) Antagonistic role of E4BP4 and PAR proteins in the circadian oscillatory mechanism. Genes Dev 15: 995-1006.

39. Ohno T, Onishi Y, Ishida N (2007) The negative transcription factor E4BP4 is associated with circadian clock protein PERIOD2. Biochem Biophys Res Commun 354: 1010-1015.

40. Ohno T, Onishi Y, Ishida N (2007) A novel E4BP4 element drives circadian expression of mPeriod2. Nucleic Acids Res 35: 648-655.

41. Honma S, Kawamoto T, Takagi Y, Fujimoto K, Sato F, et al. (2002) Decl and Dec2 are regulators of the mammalian molecular clock. Nature 419: 841-844.

42. Matsumoto A, Ukai-Tadenuma M, Yamada RG, Houl J, Uno KD, et al. (2007) A functional genomics strategy reveals clockwork orange as a transcriptional regulator in the Drosophila circadian clock. Genes Dev 21: 1687-1700.

43. Kadener S, Stoleru D, McDonald M, Nawathean P, Rosbash M (2007) Clockwork Orange is a transcriptional repressor and a new Drosophila circadian pacemaker component. Genes Dev 21: 1675-1686.

44. Lim C, Chung BY, Pitman JL, McGill JJ, Pradhan S, et al. (2007) Clockwork orange encodes a transcriptional repressor important for circadian-clock amplitude in Drosophila. Curr Biol 17: 1082-1089.

45. Oishi K, Shirai H, Ishida N (2005) CLOCK is involved in the circadian transactivation of peroxisome-proliferator-activated receptor alpha (PPARalpha) in mice. Biochem J 386: 575-581.

46. Inoue I, Shinoda Y, Ikeda M, Hayashi K, Kanazawa K, et al. (2005) CLOCK/ BMAL1 is involved in lipid metabolism via transactivation of the peroxisome proliferator-activated receptor (PPAR) response element. J Atheroscler Thromb 12: $169-174$.

47. Canaple L, Rambaud J, Dkhissi-Benyahya O, Rayet B, Tan NS, et al. (2006) Reciprocal regulation of brain and muscle Arnt-like protein 1 and peroxisome proliferator-activated receptor alpha defines a novel positive feedback loop in the rodent liver circadian clock. Mol Endocrinol 20: 1715-1727.

48. Liu C, Li S, Liu T, Borjigin J, Lin JD (2007) Transcriptional coactivator PGClalpha integrates the mammalian clock and energy metabolism. Nature 447: $477-481$.

49. DeBruyne JP, Weaver DR, Reppert SM (2007) Peripheral circadian oscillators require CLOCK. Curr Biol 17: R538-R539.

50. Gachon F, Fonjallaz P, Damiola F, Gos P, Kodama T, et al. (2004) The loss of circadian PAR bZip transcription factors results in epilepsy. Genes Dev 18: 1397-1412.

51. Grechez-Cassiau A, Panda S, Lacoche S, Teboul M, Azmi S, et al. (2004) The transcriptional repressor STRA13 regulates a subset of peripheral circadian outputs. J Biol Chem 279: 1141-1150.

52. Takahashi JS (2004) Finding new clock components: past and future. J Biol Rhythms 19: 339-347.

53. Storch KF, Lipan O, Leykin I, Viswanathan N, Davis FC, et al. (2002) Extensive and divergent circadian gene expression in liver and heart. Nature 417: 78-83.
54. Lowrey PL, Takahashi JS (2004) Mammalian circadian biology: elucidating genome-wide levels of temporal organization. Annu Rev Genomics Hum Genet 5: 407-441.

55. Gachon F, Fleury-Olela F, Schaad O, Descombes P, Schibler U (2006) The circadian PAR-domain basic leucine zipper transcription factors DBP, TEF, and HLF modulate basal and inducible xenobiotic detoxcification. Cell Metab 4: 25-36.

56. Boukhtouche F, Mariani J, Tedgui A (2004) The "CholesteROR" protective pathway in the vascular system. Arterioscler Thromb Vasc Biol 24: 637-643.

57. Delerive P, Monte D, Dubois G, Trottein F, Fruchart-Najib J, et al. (2001) The orphan nuclear receptor ROR alpha is a negative regulator of the inflammatory response. EMBO Rep 2: 42-48.

58. Jetten AM, Kurebayashi S, Ueda E (2001) The ROR nuclear orphan receptor subfamily: Critical regulators of multiple biological processes. Progr Nucleic Acid Res Mol Biol 69: 205-247.

59. Littman DR, Sun Z, Unutmaz D, Sunshine MJ, Petrie HT, et al. (2003) The role of the nuclear hormone receptor ROR gamma in the development of lymph nodes and Peyer's patches. Immunol Rev 195: 81-90.

60. Fontaine C, Staels B (2007) The orphan nuclear receptor Rev-erbalpha: a transcriptional link between circadian rhythmicity and cardiometabolic disease. Curr Opin Lipidol 18: 141-146.

61. Kang HS, Angers M, Beak JY, Wu X, Gimble JM, et al. (2007) Gene expression profiling reveals a regulatory role for $\operatorname{ROR}\{$ alpha $\}$ and $\operatorname{ROR}\{$ gamma $\}$ in Phase I and Phase II Metabolism. Physiol Genomics 31: 281-294.

62. Lau P, Nixon SJ, Parton RG, Muscat GE (2004) RORalpha regulates the expression of genes involved in lipid homeostasis in skeletal muscle cells: caveolin-3 and CPT-1 are direct targets of ROR. J Biol Chem 279: 36828-36840.

63. Ramakrishnan SN, Lau P, Burke LJ, Muscat GE (2005) Rev-erbbeta regulates the expression of genes involved in lipid absorption in skeletal muscle cells: evidence for cross-talk between orphan nuclear receptors and myokines. J Biol Chem 280: 8651-8659.

64. Wang J, Yin L, Lazar MA (2006) The orphan nuclear receptor Rev-erb alpha regulates circadian expression of plasminogen activator inhibitor type 1 . J Biol Chem 281: 33842-33848.

65. Zvonic S, Ptitsyn AA, Conrad SA, Scott LK, Floyd ZE, et al. (2006) Characterization of peripheral circadian clocks in adipose tissues. Diabetes 55: 962-970.

66. Yoo SH, Yamazaki S, Lowrey PL, Shimomura K, Ko CH, et al, (2004) PERIOD2::LUCIFERASE real-time reporting of circadian dynamics reveals persistent circadian oscillations in mouse peripheral tissues. Proc Natl Acad Sci U S A 101: 5339-5346.

67. Welsh DK, Yoo SH, Liu AC, Takahashi JS, Kay SA (2004) Bioluminescence imaging of individual fibroblasts reveals persistent, independently phased circadian rhythms of clock gene expression. Curr Biol 14: 2289-2295.

68. Amicone L, Spagnoli FM, Spath G, Giordano S, Tommasini C, et al. (1997) Transgenic expression in the liver of truncated Met blocks apoptosis and permits immortalization of hepatocytes. EMBO J 16: 495-503.

69. Rubinson DA, Dillon CP, Kwiatkowski AV, Sievers C, Yang LL, et al. (2003) A lentivirus-based system to functionally silence genes in primary mammalian cells, stem cells and transgenic mice by RNA interference. Nat Genet 33: 401-406. 